\title{
Etch Rates for Micromachining Processing-Part II
}

\author{
Kirt R. Williams, Senior Member, IEEE, Kishan Gupta, Student Member, IEEE, and Matthew Wasilik
}

\begin{abstract}
Samples of 53 materials that are used or potentially can be used or in the fabrication of microelectromechanical systems and integrated circuits were prepared: single-crystal silicon with two doping levels, polycrystalline silicon with two doping levels, polycrystalline germanium, polycrystalline SiGe, graphite, fused quartz, Pyrex 7740, nine other preparations of silicon dioxide, four preparations of silicon nitride, sapphire, two preparations of aluminum oxide, aluminum, $\mathrm{Al} / 2 \% \mathrm{Si}$, titanium, vanadium, niobium, two preparations of tantalum, two preparations of chromium, $\mathrm{Cr}$ on $\mathrm{Au}$, molybdenum, tungsten, nickel, palladium, platinum, copper, silver, gold, $10 \mathrm{Ti} / 90 \mathrm{~W}, 80$ $\mathrm{Ni} / 20 \mathrm{Cr}$, TiN, four types of photoresist, resist pen, Parylene-C, and spin-on polyimide. Selected samples were etched in 35 different etches: isotropic silicon etchant, potassium hydroxide, 10:1 HF, 5:1 BHF, Pad Etch 4, hot phosphoric acid, Aluminum Etchant Type A, titanium wet etchant, CR-7 chromium etchant, CR-14 chromium etchant, molybdenum etchant, warm hydrogen peroxide, Copper Etchant Type CE-200, Copper Etchant APS 100, dilute aqua regia, AU-5 gold etchant, Nichrome Etchant TFN, hot sulfuric + phosphoric acids, Piranha, Microstrip 2001, acetone, methanol, isopropanol, xenon difluoride, $\mathrm{HF}+\mathrm{H}_{2} \mathrm{O}$ vapor, oxygen plasma, two deep reactive ion etch recipes with two different types of wafer clamping, $\mathrm{SF}_{6}$ plasma, $\mathrm{SF}_{6}+\mathrm{O}_{2}$ plasma, $\mathrm{CF}_{4}$ plasma, $\mathrm{CF}_{4}+\mathrm{O}_{2}$ plasma, and argon ion milling. The etch rates of 620 combinations of these were measured. The etch rates of thermal oxide in different dilutions of HF and BHF are also reported. Sample preparation and information about the etches is given.

[1070]
\end{abstract}

Index Terms-Chemical vapor deposition (CVD), etching, evaporation, fabrication, materials processing, micromachining.

\section{INTRODUCTION}

W HEN designing a microfabrication process, the etch rate of each material to be etched must be known. Knowing the etch rates of other materials that will be exposed to the etch, such as masking films and underlying layers, enables an etch process to be chosen for good selectivity (high ratio of etch rate of the target material to etch rate of the other material)-if one exists. While several large literature-review compilations of etches that target specific materials have been made [1], [2], these only report etch rates in some cases, and rarely have corresponding selectivity information. This paper provides such information, expanding on an earlier paper [3] to give 620 etch rates of 53 materials in 35 etches that have been used or may

Manuscript received June 3, 2003; revised October 1, 2003. Subject Editor A. J. Ricco.

K. R. Williams was with Agilent Laboratories, Agilent Technologies, Palo Alto, 94303 CA USA. He is currently a private consultant at 185 Willowbrook Dr., Portola Valley, CA 94028 USA (e-mail: kirt_williams@ @ieee.org).

K. Gupta was with with Agilent Laboratories, Agilent Technologies, Palo Alto, CA 94303 USA. He is now at 804 Gregory Ct., Fremont, CA 94359 USA (e-mail: kishang@ieee.org).

M. Wasilik is with the Berkeley Sensor \& Actuator Center, University of California at Berkeley, Berkeley, CA 94720-1770 USA.

Digital Object Identifier 10.1109/JMEMS.2003.820936 be used in future fabrication of microelectromechanical systems (MEMS) and integrated circuits (ICs) (approximately 50 etch rates measured in the earlier paper have been included in this one). These data allow the selection of new combinations of structural material, underlying material, and etchant for micromachining.

Table I summarizes the etches tested, abbreviated names for the etches, and the target materials for each. Table II lists etch rates of $\mathrm{Si}, \mathrm{Ge}, \mathrm{SiGe}$, and $\mathrm{C}$ in the SI units of $\mathrm{nm} / \mathrm{min}$ (not $\AA / \mathrm{min}$ as in the earlier tables) [3]. Table III covers films and wafers that are primarily silicon dioxide, produced under many different conditions. Table IV is on silicon nitride and aluminum oxide. Table V covers the metals Al, Ti, V, Nb, Ta, and Cr. Table VI continues with the metals $\mathrm{Mo}, \mathrm{W}, \mathrm{Ni}, \mathrm{Pd}, \mathrm{Pt}, \mathrm{Cu}, \mathrm{Ag}, \mathrm{Au}$, alloys $10 \mathrm{Ti} / 90 \mathrm{~W}, 80 \mathrm{Ni} / 20 \mathrm{Cr}$, and compound TiN. Finally, Table VII gives etch rates of organics: photoresists, a resist pen, and a spin-on polyimide.

Section II of this paper lists the materials etched, their preparation, and some uses or potential uses in MEMS and ICs. Section III describes the preparation and applications of the wet and dry etches that were studied, as well as some key experimental results. Section IV describes etch-rate measurement techniques, and Section V discusses the results.

\section{SAMPLE PREPARATION}

The preparation of the samples in the etch-rate tables is described below, listed by the labels (in italics) used across the tops of the tables. All coated materials were deposited on 100-mmdiameter silicon wafers. For the isotropic silicon etchant, potassium hydroxide, and a few other etches, the wafers were first coated with LPCVD silicon nitride so that etches would not penetrate into the silicon or attack the back side of the wafer.

In several cases, similar materials were prepared using different methods (e.g., wafer form, PECVD, LPCVD, and ionmilled silicon dioxide; annealed and unannealed films) to study and emphasize the effect on their etching characteristics.

Existing or potential MEMS applications are given for the materials. Many of the materials were discussed in more detail previously [3].

\section{A. Silicon, Germanium, SiGe, and Carbon}

(100) Si Low-Doped Wafer: Single-crystal silicon, (100) orientation, phosphorus-doped n-type, resistivity of 3-40 $\Omega$-cm, grown with the Czochralski (CZ method). Single-crystal silicon is the standard starting material for bulk micromachining.

Float-Zone Si Wafer: Single-crystal silicon, (100) orientation, undoped, grown with the float-zone (FZ) method for a high resistivity of $>10000 \Omega$-cm. Float-zone wafers have been used as substrates in RF MEMS application to reduce eddy-current loss. 
TABLE I

ETCH DESCRIPTIONS, ABBREVIATIONS, AND TARGET Materials

\begin{tabular}{|c|c|c|}
\hline Etchant & $\begin{array}{l}\text { Etchant } \\
\text { Abbrev. }\end{array}$ & $\begin{array}{c}\text { Target } \\
\text { Material } \\
\end{array}$ \\
\hline Isotropic Silicon Etchant "Trilogy Etch" (126 $\left.\mathrm{HNO}_{3}: 60 \mathrm{H}_{2} \mathrm{O}: 5 \mathrm{NH}_{4} \mathrm{~F}\right), \sim 20^{\circ} \mathrm{C}$ & Si Iso Etch & Silicon \\
\hline $\mathrm{KOH}\left(30 \%\right.$ by weight), $80^{\circ} \mathrm{C}$ & $\mathrm{KOH}$ & Silicon ODE \\
\hline $10: 1 \mathrm{HF}\left(10 \mathrm{H}_{2} \mathrm{O}: 149 \% \mathrm{HF}\right),-20^{\circ} \mathrm{C}$ & $10: 1 \mathrm{HF}$ & Silicon Dioxide \\
\hline $5: 1 \mathrm{BHF}\left(540 \% \mathrm{NH}_{4} \mathrm{~F}: 149 \% \mathrm{HF}\right), \sim 20^{\circ} \mathrm{C}$ & $5: 1 \mathrm{BHF}$ & Silicon Dioxide \\
\hline Pad Etch 4 from Ashland $\left(13 \% \mathrm{NH}_{4} \mathrm{~F}+32 \% \mathrm{HAC}+49 \% \mathrm{H}_{2} \mathrm{O}+6 \%\right.$ propylene glycol + surfactant), $20^{\circ} \mathrm{C}$ & Pad Etch 4 & $\mathrm{SiO}_{2}$, not Al \\
\hline Phosphoric Acid ( $85 \%$ by weight), $160^{\circ} \mathrm{C}$ & Phosphoric & Silicon nitride \\
\hline Al Etchant Type A from Transene $\left(80 \% \mathrm{H}_{3} \mathrm{PO}_{4}+5 \% \mathrm{HNO}_{3}+5 \% \mathrm{HAC}+10 \% \mathrm{H}_{2} \mathrm{O}\right), 50 \circ \mathrm{C}$ & Al Etch A & Aluminum \\
\hline Titanium wet etchant $\left(20 \mathrm{H}_{2} \mathrm{O}: 1 \mathrm{H}_{2} \mathrm{O}_{2}: 1 \mathrm{HF}\right),-20^{\circ} \mathrm{C}$ & Ti Etch & Titanium \\
\hline Chromium etchant CR-7 from Cyantek $\left.\left(9 \%\left(\mathrm{NH}_{4}\right)_{2} \mathrm{Ce}\left(\mathrm{NO}_{3}\right)_{6}\right)+6 \% \mathrm{HClO}_{4}+\mathrm{H}_{2} \mathrm{O}\right), \sim 20^{\circ} \mathrm{C}$ & CR-7 & Chromium \\
\hline Chromium etchant CR-14 from Cyantek $\left.\left(22 \%(\mathrm{NH} 4)_{2} \mathrm{Ce}(\mathrm{NO} 3)_{6}\right)+8 \% \mathrm{HAC}+\mathrm{H} 2 \mathrm{O}\right), \sim 20^{\circ} \mathrm{C}$ & CR-14 & Chromium \\
\hline Molybdenum etchant $\left(180 \mathrm{H}_{3} \mathrm{PO}_{4}: 11 \mathrm{HAC}: 11 \mathrm{HNO}_{3}: 150 \mathrm{H}_{2} \mathrm{O}\right), \sim 20^{\circ} \mathrm{C}$ & Moly Etch & Molybdenum \\
\hline Hydrogen peroxide $\left(30 \mathrm{w} t \% \mathrm{H}_{2} \mathrm{O}_{2}, 70 \mathrm{wt} \% \mathrm{H}_{2} \mathrm{O}\right), 50^{\circ} \mathrm{C}$ & $\mathrm{H}_{2} \mathrm{O}_{2} 50^{\circ} \mathrm{C}$ & Tungsten \\
\hline Copper etchant type CE-200 from Transene $\left(30 \% \mathrm{FeCl}_{3}+3-4 \% \mathrm{HCl}+\mathrm{H}_{2} \mathrm{O}\right),-20^{\circ} \mathrm{C}$ & $\mathrm{Cu} \mathrm{FeCl}{ }_{3} 200$ & Copper \\
\hline Copper etchant APS 100 from Transene $\left(15-20 \%\left(\mathrm{NH}_{4}\right)_{2} \mathrm{~S}_{2} \mathrm{O}_{8}+\mathrm{H}_{2} \mathrm{O}\right), 30^{\circ} \mathrm{C}$ & Cu APS 100 & Copper \\
\hline Dilute aqua regia $\left(3 \mathrm{HCl}: 1 \mathrm{HNO}_{3}: 2 \mathrm{H}_{2} \mathrm{O}\right),-30^{\circ} \mathrm{C}$ & Dil. Aqua regia & Noble metals \\
\hline Gold etchant AU-5 from Cyantek $\left(5 \% \mathrm{I}_{2}+10 \% \mathrm{KI}+85 \% \mathrm{H}_{2} \mathrm{O}\right), \sim 20^{\circ} \mathrm{C}$ & AU-5 & Gold \\
\hline Nichrome etchant TFN from Transene $\left.\left(10-20 \%\left(\mathrm{NH}_{4}\right)_{2} \mathrm{Ce}\left(\mathrm{NO}_{3}\right)_{6}\right)+5-6 \% \mathrm{HNO}_{3}+\mathrm{H}_{2} \mathrm{O}\right),-20^{\circ} \mathrm{C}$ & NiCr TFN & $\mathrm{NiCr}$ \\
\hline $1 \mathrm{H}_{2} \mathrm{SO}_{4}: 1 \mathrm{H}_{3} \mathrm{PO}_{4}, 160^{\circ} \mathrm{C}$ & Phos+Sulf & Sapphire \\
\hline Piranha $\left(-50 \mathrm{H}_{2} \mathrm{SO} 4: 1 \mathrm{H}_{2} \mathrm{O}_{2}\right), 120^{\circ} \mathrm{C}$ & Piranha & Cleaning \\
\hline Microstrip 2001 photoresist stripper, $85^{\circ} \mathrm{C}$ & Microstrip & Photoresist \\
\hline Acetone, $-20^{\circ} \mathrm{C}$ & Acetone & Photoresist \\
\hline Methanol, $-20^{\circ} \mathrm{C}$ & Methanol & Cleaning \\
\hline Isopropanol, $-20^{\circ} \mathrm{C}$ & IPA & Cleaning \\
\hline $\mathrm{XeF}_{2}, 2.6$ mTorr, homemade chamber & $\mathrm{XeF}_{2}$ & Silicon \\
\hline $\mathrm{HF}+\mathrm{H}_{2} \mathrm{O}$ vapor, $1 \mathrm{~cm}$ over dish with $49 \% \mathrm{HF}$ & HF vapor & Silicon dioxide \\
\hline Technics plasma, $\mathrm{O}_{2}, 400 \mathrm{~W} @ 30 \mathrm{kHz}, 300 \mathrm{mTorr}$ & Technics $\mathrm{O}_{2}$ & Photoresist \\
\hline STS ASE DRIE, mechanical chuck, high frequency, typical recipe & DRIE HF mech. & Silicon \\
\hline STS ASE DRIE, electrostatic chuck, high frequency, typical recipe & DRIE HF ES & Silicon \\
\hline STS ASE DRIE, mechanical chuck, stop-on-oxide (low-frequency platen), typical recipe & DRIE LF mech. & Silicon \\
\hline STS ASE DRIE, electrostatic chuck, stop-on-oxide (low-frequency platen), typical recipe & DRIE LF ES & Silicon \\
\hline STS 320 RIE, SF $6,100 \mathrm{~W} @ 13.56 \mathrm{MHz}, 20 \mathrm{mTorr}$ & STS $320 \mathrm{SF}_{6}$ & $\mathrm{Si}, \mathrm{SiN}$, metals \\
\hline STS 320 RIE, $\mathrm{SF}_{6}+\mathrm{O}_{2}, 100 \mathrm{~W} @ 13.56 \mathrm{MHz}, 20 \mathrm{mTorr}$ & $\mathrm{STS} \mathrm{SF} \mathrm{F}_{6}+\mathrm{O}_{2}$ & $\mathrm{Si}, \mathrm{SiN}$, metals \\
\hline STS 320 RIE, $\mathrm{CF}_{4}, 100 \mathrm{~W} @ 13.56 \mathrm{MHz}, 60 \mathrm{mTorr}$ & STS $320 \mathrm{CF}_{4}$ & $\mathrm{Si}, \mathrm{SiO}, \mathrm{SiN}$ \\
\hline STS 320 RIE, $\mathrm{CF}_{4}+\mathrm{O}_{2}, 100 \mathrm{~W} @ 13.56 \mathrm{MHz}, 60 \mathrm{mTorr}$ & STS $\mathrm{CF}_{4}+\mathrm{O}_{2}$ & $\mathrm{Si}, \mathrm{SiO}, \mathrm{SiN}$ \\
\hline Ion milling with argon ions at $500 \mathrm{~V}, \sim 1 \mathrm{~mA} \mathrm{~cm}^{2}$, normal incidence (Commonwealth data) & Ion Mill & Everything \\
\hline
\end{tabular}

Polysilicon LPCVD Undoped: Undoped polycrystalline silicon deposited in a Tylan low-pressure chemical-vapor deposition (LPCVD) furnace with recipe $\mathrm{SiH}_{4}$ flow rate $=100 \mathrm{sccm}$, temperature $(\mathrm{T})=605{ }^{\circ} \mathrm{C}$, pressure $(\mathrm{p})=300$ mtorr. Deposited on a wafer with $100 \mathrm{~nm}$ of thermal oxide on it to enable interferometric thickness measurements. Refractive index $(\mathrm{RI})=3.97$. Undoped poly, which has a high sheet resistance as deposited, is the most common structural material for surface micromachining. It can be doped with ion implantation or by diffusing in dopant atoms from an adjacent film (e.g., PSG, below) at high temperature.
Polysilicon LPCVD In-Situ $\mathrm{n}^{+}$: An n-type, phosphorusdoped polycrystalline silicon deposited in a Tylan LPCVD furnace with recipe $\mathrm{SiH}_{4}=100 \mathrm{sccm}, 1.6 \% \mathrm{PH}_{3} /$ balance $\mathrm{SiH}_{4}=2 \mathrm{sccm}, \mathrm{T}=610^{\circ} \mathrm{C}, \mathrm{p}=300$ mtorr. Deposited on a wafer with thermal oxide on it to enable interferometric thickness measurements. RI $=3.97$. In situ doping gives a conducting film, useful for thicker films and in cases in which other considerations limit the temperature. The deposition rate is about $1 / 3$ that of undoped polysilicon under similar conditions.

Poly Ge LPCVD Undoped: Undoped polycrystalline germanium deposited in Tystar LPCVD furnace with recipe $\mathrm{GeH}_{4}=$ 
TABLE II

ETCH RATES OF $\mathrm{Si}, \mathrm{Ge}, \mathrm{SiGe}$, AND C (nm/min)

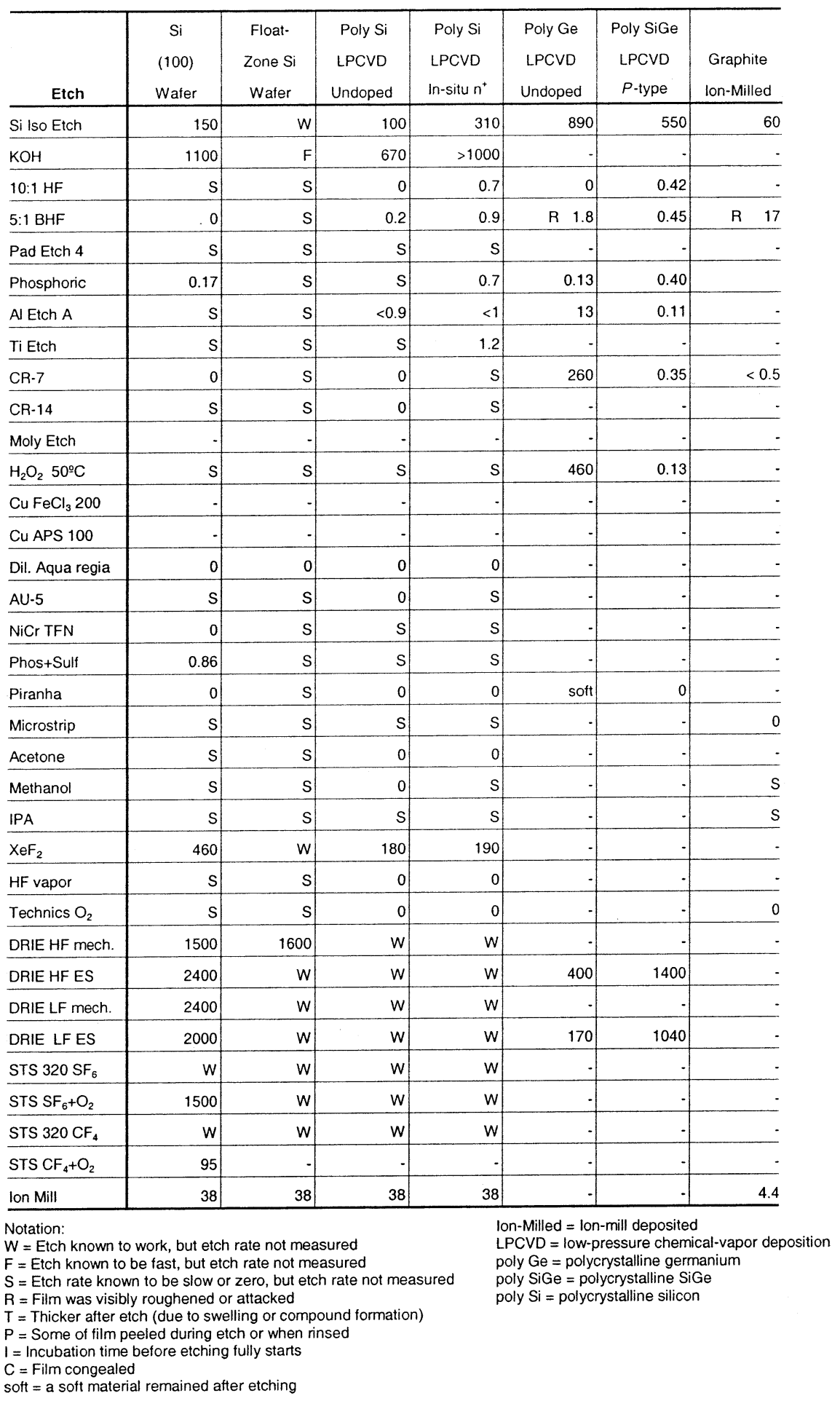

$100 \mathrm{sccm}, \mathrm{T}=400{ }^{\circ} \mathrm{C}, \mathrm{p}=300$ mtorr. The polygermanium deposition was preceded by the deposition of silicon seed layer approximately $6 \mathrm{~nm}$ thick using the recipe $\mathrm{Si}_{2} \mathrm{H}_{6}=100 \mathrm{sccm}$, $\mathrm{T}=400{ }^{\circ} \mathrm{C}, \mathrm{p}=300$ mtorr.

Germanium forms an oxide that is soluble in water. Thus, water with a high concentration of dissolved oxygen etches germanium. Hydrogen peroxide is a useful etchant for $\mathrm{Ge}$, etching faster at higher temperature.
Polygermanium has been used in surface micromachining as a sacrificial layer in conjunction with a polycrystalline SiGe structural layer, using warm hydrogen peroxide as the etchant [4]. The relatively low deposition temperatures are compatible with CMOS circuitry with aluminum interconnections.

Poly SiGe LPCVD p-Type: A p-type polycrystalline silicongermanium deposited in a Tystar LPCVD furnace with recipe $\mathrm{SiH}_{4}=100 \mathrm{sccm}, \mathrm{GeH}_{4}=58 \mathrm{sccm}, \mathrm{B}_{2} \mathrm{H}_{6}=60 \mathrm{sccm}, \mathrm{T}=$ 
TABLE III

ETCH RATES OF SILICON DIOXIDE (nm/min)

\begin{tabular}{|c|c|c|c|c|c|c|c|c|c|c|c|}
\hline Etch & $\begin{array}{l}\text { Fused } \\
\text { Quartz } \\
\text { Wafer } \\
\end{array}$ & $\begin{array}{c}\text { Pyrex } \\
7740 \\
\text { Wafer } \\
\end{array}$ & $\begin{array}{c}\text { Thermal } \\
\text { Oxide } \\
\text { Wet-Grn }\end{array}$ & $\begin{array}{l}\text { Ann. LTO } \\
\text { LPCVD } \\
\text { Calogic } \\
\end{array}$ & $\begin{array}{c}\text { Unan. LTO } \\
\text { LPCVD } \\
\text { Tylan } \\
\end{array}$ & $\begin{array}{l}\text { Ann. LTO } \\
\text { LPCVD } \\
\text { Tylan }\end{array}$ & $\begin{array}{c}\text { Unan. PSG } \\
\text { LPCVD } \\
\text { Tylan } \\
\end{array}$ & $\begin{array}{c}\text { Ann. PSG } \\
\text { LPCVD } \\
\text { Tylan } \\
\end{array}$ & $\begin{array}{c}\text { Oxide } \\
\text { PECVD } \\
\text { Unannealed } \\
\end{array}$ & $\begin{array}{c}\text { Oxide } \\
\text { PECVD } \\
\text { Annealed } \\
\end{array}$ & $\begin{array}{c}\text { Oxide } \\
\text { Ion-Mill }\end{array}$ \\
\hline Si Iso Etch & 12 & R 140 & 8.7 & 15 & & 11 & 400 & 170 & 100 & 25 & 43 \\
\hline $\mathrm{KOH}$ & 6.7 & 11 & 7.7 & 8.1 & & 9.4 & & 38 & 15 & 7.8 & 8.0 \\
\hline 10:1 HF & 26 & $w$ & 23 & $w$ & $w$ & 34 & 1500 & 470 & $w$ & $w$ & W \\
\hline 5:1 BHF & 130 & 43 & 100 & 150 & w & 120 & 680 & 440 & 490 & 240 & 82 \\
\hline Pad Etch 4 & 29 & 17 & 31 & w & 38 & $w$ & 200 & $w$ & 160 & $w$ & w \\
\hline Phosphoric & 0.23 & 3.7 & 0.18 & $\mathrm{~s}$ & 0.21 & 0.21 & 2.7 & 1.8 & - & - & $\mathrm{S}$ \\
\hline Al Etch A & $\mathrm{s}$ & - & 0 & $s$ & $\mathrm{~s}$ & 0 & $\mathrm{~s}$ & $<1$ & 0 & s & $S$ \\
\hline Ti Etch & - & - & 12 & $w$ & $w$ & $w$ & $w$ & 210 & $w$ & $w$ & - \\
\hline CR-7 & $R<0.4$ & $\begin{array}{ll}R & 0\end{array}$ & 0.02 & 0 & $\mathrm{~s}$ & $s$ & $\mathrm{~s}$ & s & 0 & 0 & 0 \\
\hline CR-14 & $\mathrm{s}$ & - & 0.01 & $\mathrm{~s}$ & $\mathrm{~s}$ & $\mathrm{~s}$ & $\mathrm{~s}$ & $\mathrm{~s}$ & s & $\mathrm{s}$ & $\mathrm{s}$ \\
\hline Moly Etch & - & . & - & -1 & - & - & - & - & - & - & - \\
\hline $\mathrm{H}_{2} \mathrm{O}_{2} \quad 50^{\circ} \mathrm{C}$ & $\mathrm{s}$ & $s$ & 0 & $\mathrm{~s}$ & $s$ & $\mathrm{~s}$ & $\mathrm{~s}$ & $s$ & $\mathrm{~s}$ & $\mathrm{~s}$ & $S$ \\
\hline $\mathrm{Cu} \mathrm{FeCl} 2200$ & - & . & - & - & - & - & - & - & & - & - \\
\hline Cu APS 100 & - & - & - & - & - & . & - & - & - & - & - \\
\hline Dil. Aqua regia & 0 & - & 0 & 0 & 0 & 0 & 0 & 0 & 0.7 & s & $\mathrm{S}$ \\
\hline AU-5 & $\mathrm{s}$ & - & $\mathrm{s}$ & $\mathrm{s}$ & $\mathrm{s}$ & $\mathrm{s}$ & $\mathrm{s}$ & $\mathrm{s}$ & 0 & $\mathrm{~s}$ & $\mathrm{~s}$ \\
\hline $\mathrm{NiCr}$ TFN & $\mathrm{s}$ & - & $\mathrm{s}$ & $\mathrm{s}$ & $\mathrm{s}$ & $\mathrm{s}$ & $\mathrm{s}$ & $\mathrm{s}$ & $\mathrm{s}$ & $\mathrm{s}$ & $\mathrm{S}$ \\
\hline Phos+Sulf & $\mathrm{s}$ & - & 0.057 & $\mathrm{~s}$ & $\mathrm{~s}$ & s) & $\mathrm{s}$ & $\mathrm{s}$ & $\mathrm{s}$ & $\mathrm{s}$ & $\mathrm{S}$ \\
\hline Piranha & $\begin{array}{ll}R & 0 \\
\end{array}$ & R 0 & 0 & 0 & $\mathrm{~s}$ & 0 & $\mathrm{~s}$ & 0 & 0 & 0 & 0 \\
\hline Microstrip & $\mathrm{s}$ & $\mathrm{s}$ & $\mathrm{s}$ & $\mathrm{s}$ & $\mathrm{s}$ & $\mathrm{s}$ & $\mathrm{s}$ & $\mathrm{s}$ & $\mathrm{s}$ & $\mathrm{s}$ & $\mathrm{S}$ \\
\hline Acetone & $\mathrm{s}$ & $\mathrm{s}$ & 0 & $\mathrm{~s}$ & $\mathrm{~s}$ & 0 & $\mathrm{~s}$ & 0 & $\mathrm{~s}$ & $\mathrm{~s}$ & $\mathrm{~S}$ \\
\hline Methanol & $\mathrm{s}$ & $\mathrm{s}$ & $\mathrm{s}$ & $\mathrm{s}$ & $\mathrm{s}$ & $\mathrm{s}$ & $\mathrm{s}$ & $\mathrm{s}$ & $\mathrm{s}$ & $\mathrm{s}$ & $\mathrm{S}$ \\
\hline IPA & $\mathrm{s}$ & $\mathrm{s}$ & $\mathrm{s}$ & $\mathrm{s}$ & s) & s & $\mathrm{s}$ & $\mathrm{s}$ & $\mathrm{s}$ & s) & $\mathrm{s}$ \\
\hline $\mathrm{XeF}_{2}$ & $\mathrm{~s}$ & - & 0 & $\mathrm{~s}$ & s) & 0 & 0 & 0 & $\mathrm{~s}$ & $\mathrm{~s}$ & $\mathrm{~s}$ \\
\hline HF vapor & $w$ & $w$ & 66 & W & w & 78 & 210 & 150 & w & w & W \\
\hline Technics $\mathrm{O}_{2}$ & $\mathrm{~s}$ & $\mathrm{~s}$ & 0 & $\mathrm{~s}$ & s) & 0 & 0 & 0 & $\mathrm{~s}$ & $\mathrm{~s}$ & $\mathrm{~S}$ \\
\hline DRIE HF mech. & $\mathrm{s}$ & - & $\mathrm{s}$ & 7.5 & 6.2 & 6.9 & 9.5 & 11 & 9.5 & $\mathrm{~s}$ & S \\
\hline DRIE HF ES & $\mathrm{s}$ & - & 24 & $w$ & $w$ & w & w & w & $w$ & $w$ & $w$ \\
\hline DRIE LF mech. & $\mathrm{s}$ & - & $\mathrm{s}$ & 3.6 & 9.8 & 9.4 & 15 & 15 & 4.0 & $\mathrm{~s}$ & $\mathrm{~S}$ \\
\hline DRIE LFES & $\mathrm{s}$ & - & 24 & w & $w$ & w & w & w & w & w & W \\
\hline STS $320 \mathrm{SF}_{6}$ & w & - & $w$ & $w$ & w & w & $w$ & w & $w$ & $w$ & w \\
\hline STS SF ${ }_{6}+\mathrm{O}_{2}$ & 35 & 10 & 29 & 38 & 55 & 48 & 73 & 60 & 55 & 32 & 30 \\
\hline STS $320 \mathrm{CF}_{4}$ & $w$ & $w$ & $w$ & 33 & $w$ & $w$ & w & W & W & w & w \\
\hline $\mathrm{STS} \mathrm{CF}_{4}+\mathrm{O}_{2}$ & 41 & 31 & 44 & 42 & 51 & 46 & 69 & 62 & 51 & 43 & 21 \\
\hline Ion Mill & w & $w$ & 39 & $w$ & $w$ & $w$ & $w$ & $w$ & $w$ & w & W \\
\hline \multicolumn{5}{|c|}{$\begin{array}{l}\text { Notation: } \\
W=\text { Etch known to work, but etch rate not measured } \\
F=\text { Etch known to be fast, but etch rate not measured } \\
S=\text { Etch rate known to be slow or zero, but etch rate not measured } \\
R=\text { Film was visibly roughened or attacked } \\
T=\text { Thicker after etch (due to swelling or compound formation) } \\
P=\text { Some of film peeled during etch or when rinsed } \\
I=\text { Incubation time before etching fully starts } \\
C=\text { Film congealed } \\
\text { soft = a soft material remained after etching }\end{array}$} & \multicolumn{7}{|c|}{$\begin{array}{l}\text { Ann. LTO = annealed low-temperature oxide } \\
\text { Ann. PSG = annealed phosphosilicate glass } \\
\text { LPCVD }=\text { low-pressure chemical vapor deposition } \\
\text { PECVD = plasma-enhance chemical-vapor deposition } \\
\text { Unan. LTO = unannealed low-temperature oxide } \\
\text { Unan. PSG = unannealed phosphosilicate glass }\end{array}$} \\
\hline
\end{tabular}

$400{ }^{\circ} \mathrm{C}, \mathrm{p}=300$ mtorr. This film is approximately 48 atomic $\% \mathrm{Ge}$.

Graphite Ion-Milled: Graphite ion-mill-deposited (also known as ion-beam-deposited) in a Commonwealth Scientific system from a graphite target with argon ions at $1250 \mathrm{~V}$, current density of about $2 \mathrm{~mA} / \mathrm{cm}^{2}(\sim 200 \mathrm{~mA}$ over most of a 5-inch-diameter target), chamber $\mathrm{p}=3 \times 10^{-4}$ torr. Graphite has had little or no use in MEMS to date. In this work, it was found to be easily deposited and etched in silicon isotropic etchant. It may find use as a hard mask for plasma etching due to its low etch rate, and as a dry lubricant in MEMS.

\section{B. Silicon Dioxide}

Fused Quartz Wafer: Wafers of General Electric 124 or NSG N fused quartz source material, $>99.99 \%$ silicon dioxide, with amorphous structure (as opposed to true crystalline quartz). This material is commonly referred to simply as "quartz." It is compatible with silicon-wafer processing steps, and may find application as a substrate in RF MEMS as it is not conductive, eliminating eddy-current losses.

Pyrex 7740 Wafer: Corning Pyrex 7740 glass, $81 \% \mathrm{SiO}_{2}$, $13 \% \mathrm{~B}_{2} \mathrm{O}_{3}, 4 \% \mathrm{Na}_{2} \mathrm{O}, 2 \% \mathrm{Al}_{2} \mathrm{O}_{3}$. Pyrex 7740 (and the very 
TABLE IV

ETCH RATES OF SILICON NitRIDE AND ALUMINUM OXIDE (nm/min)

\begin{tabular}{|c|c|c|c|c|c|c|c|}
\hline Etch & $\begin{array}{l}\text { Stoich } \\
\text { Si Nit. } \\
\text { LPCVD } \\
\end{array}$ & $\begin{array}{l}\text { Si-Rich } \\
\text { Si Nit. } \\
\text { LPCVD }\end{array}$ & $\begin{array}{c}\text { PECVD } \\
\text { Silicon Nit. } \\
\text { Low RI } \\
\end{array}$ & $\begin{array}{c}\text { PECVD } \\
\text { Silicon Nit. } \\
\text { High RI }\end{array}$ & $\begin{array}{c}\text { Sapphire } \\
\text { Wafer }\end{array}$ & $\begin{array}{c}\text { Aluminum } \\
\text { Oxide } \\
\text { Ion-Mill } \\
\end{array}$ & $\begin{array}{c}\begin{array}{c}\text { Aluminum } \\
\text { Oxide } \\
\text { Evap }\end{array} \\
\end{array}$ \\
\hline Si Iso Etch & & 0.23 & $>66$ & 12 & 2. $R<0.7$ & 99 & 12 \\
\hline $\mathrm{KOH}$ & 0 & 0 & 0.67 & 0 & $\begin{array}{ll}R & 0\end{array}$ & $>2500$ & $>800$ \\
\hline $10: 1 \mathrm{HF}$ & 1.1 & s & & - & $\mathrm{s}$ & & - \\
\hline 5:1 BHF & s & 1.3 & 60 & 8.2 & 0 & & 160 \\
\hline Pad Etch 4 & 0.41 & $\mathrm{~s}$ & - & 1.6 & s & & $\dot{-}$ \\
\hline Phosphoric & 4.5 & 2.7 & $w$ & 20 & $<0.1$ & . & $>5$ \\
\hline Al Etch A & s & $<0.05$ & - & - & $R<2$ & 65 & 5.7 \\
\hline Ti Etch & 0.99 & s & - & - & s & $\therefore$ & - \\
\hline CR-7 & $\mathrm{s}$ & 0 & $<0.14$ & 0 & $\begin{array}{ll}R & 0\end{array}$ & 0.34 & 0.075 \\
\hline CR-14 & $\mathrm{s}$ & s & - & - & $s$ & & - \\
\hline Moly Etch & - & - & -1 & - & - & & - \\
\hline $\mathrm{H}_{2} \mathrm{O}_{2} \quad 50^{\circ} \mathrm{C}$ & 0 & s & $\mathrm{s}$ & $\mathrm{s}$ & $s$ & & - \\
\hline $\mathrm{Cu} \mathrm{FeCl}{ }_{3} 200$ & - & 0 & - & - & & & - \\
\hline Cu APS 100 & - & 0 & - & - &. & & - \\
\hline Dil. Aqua regia & 0 & 0 & - & & $s$ & & 1.1 \\
\hline $\mathrm{AU}-5$ & s) & 0 & - & - & -1 & - & - \\
\hline NiCr TFN & s & s & - & - & - & - & $\therefore$ \\
\hline Phos+Sulf & 2.9 & s & w & 10 & $<0.3$ & . & - \\
\hline Piranha & 0 & 0 & $<0.04$ & 0 & $\begin{array}{ll}R & 0\end{array}$ & 97 & 19 \\
\hline Microstrip & $\mathrm{s}$ & s & $\mathrm{s}$ & $s$ & $\mathrm{~s}$ & - & - \\
\hline Acetone & 0 & 0 & s & s & $\mathrm{s}$ & 0 & s \\
\hline Methanol & s & 0 & s & $\mathrm{s}$ & $\mathrm{s}$ & 0 & S \\
\hline IPA & s & 0. & s & s & s & 0 & S \\
\hline $\mathrm{XeF}_{2}$ & 12 & - & - & - & -1 & - & - \\
\hline HF vapor & 1.0 & 1.9 & - & - & $s$ & ( & - \\
\hline Technics $\mathrm{O}_{2}$ & 0 & $\mathrm{~s}$ & $\mathrm{~s}$ & s & $\mathrm{s}$ & $\mathrm{s}$ & s \\
\hline DRIE HF mech. & $w$ & 21 & $w$ & $w$ & $\mathrm{~s}$ & $\mathrm{~s}$ & S \\
\hline DRIE HF ES & w & $w$ & $w$ & $w$ & $\mathrm{~s}$ & $\mathrm{~s}$ & S \\
\hline DRIE LF mech. & w & 26 & $w$ & $w$ & $\mathrm{~s}$ & s & $s$ \\
\hline DRIE LFES & $w$ & w & $w$ & $w$ & $\mathrm{~s}$ & $\mathrm{~s}$ & s \\
\hline STS $320 \mathrm{SF}_{6}$ & w & w & $w$ & $w$ & $\mathrm{~s}$ & $\mathrm{~s}$ & $\mathrm{~s}$ \\
\hline STS $\mathrm{SF}_{6}+\mathrm{O}_{2}$ & 150 & 150 & 200 & 190 & 2.2 & 0.55 & 0.41 \\
\hline STS $320 \mathrm{CF}_{4}$ & 34 & w & $w$ & $w$ & s & $s$ & $\mathrm{~s}$ \\
\hline STS $\mathrm{CF}_{4}+\mathrm{O}_{2}$ & 120 & $>130$ & 240 & 110 & 0 & $<2$ & $<0.2$ \\
\hline Ion Mill & 13 & 9.4 & w & $w$ & w & 10 & 10 \\
\hline \multicolumn{5}{|c|}{$\begin{array}{l}\text { Notation: } \\
W=\text { Etch known to work, but etch rate not measured } \\
F=\text { Etch known to be fast, but etch rate not measured } \\
S=\text { Etch rate known to be slow or zero, but etch rate not measured } \\
R=\text { Film was visibly roughened or attacked } \\
T=\text { Thicker after etch (due to swelling or compound formation) } \\
P=\text { Some of film peeled during etch or when rinsed } \\
I=\text { Incubation time before etching fully starts } \\
C=\text { Film congealed } \\
\text { soft = a soft material remained after etching }\end{array}$} & \multicolumn{3}{|c|}{ tric } \\
\hline
\end{tabular}

similar Borofloat glass) are used in anodic bonding to silicon due to the high content of mobile sodium ions and to the good match of thermal expansion rates. The large amounts of nonsilicon-dioxide "impurities" give it noticeably different etching characteristics, etching slower in 5:1 BHF, but faster in silicon isotropic etchant.

Thermal Oxide Wet-Grown: Silicon dioxide grown in a Tylan atmospheric-pressure furnace with the recipe $\mathrm{O}_{2}$ carrier gas at $200 \mathrm{sccm}, \mathrm{H}_{2} \mathrm{O}$ vapor at a pressure just below $1 \mathrm{~atm}$ (the water source is at $98{ }^{\circ} \mathrm{C}$ ) at $1100{ }^{\circ} \mathrm{C}$, and a total pressure of 1 atm, followed by a $20-\min \mathrm{N}_{2}$ anneal at $1100{ }^{\circ} \mathrm{C}$. $\mathrm{RI}=1.46$. Thermal oxide forms a conformal coating on silicon. It is denser and etches more slowly than chemical-vapor-deposited oxides.

Ann. LTO LPCVD Calogic: Low-temperature silicon dioxide (LTO) deposited in a Calogic low-temperature 
TABLE V

ETCH RATES OF AL, Ti, V, Nb, Ta, AND Cr (nm/min)

\begin{tabular}{|c|c|c|c|c|c|c|c|c|c|c|}
\hline Etch & $\begin{array}{c}\text { Aluminum } \\
\text { Evap } \\
\end{array}$ & $\begin{array}{l}\mathrm{Al}+2 \% \mathrm{Si} \\
\text { Sputtered }\end{array}$ & $\begin{array}{l}\text { Titanium } \\
\text { Sputtered }\end{array}$ & $\begin{array}{c}\text { Vanadium } \\
\text { Evap } \\
\end{array}$ & $\begin{array}{l}\text { Niobium } \\
\text { Ion-Mill }\end{array}$ & $\begin{array}{c}\text { Tantalum } \\
\text { Evap } \\
\end{array}$ & $\begin{array}{l}\text { Tantalum } \\
\text { Ion-Mill } \\
\end{array}$ & $\begin{array}{c}\text { Chromium } \\
\text { Evap } \\
\end{array}$ & $\begin{array}{c}\text { Chromium } \\
\text { Ion-Mill } \\
\end{array}$ & $\begin{array}{c}\text { Patterned } \\
\text { Cr on Au } \\
\text { Evap } \\
\end{array}$ \\
\hline Si Iso Etch & 60 & 400 & 300 & 9600 & 79 & 5.8 & 5.3 & $\begin{array}{ll}\text { A } & 8.8 \\
\end{array}$ & - & $<2.3$ \\
\hline $\mathrm{KOH}$ & 12,900 & $\mathrm{~F}$ & soft & $<12$ & 3.2 & $\mathrm{~s}$ & 2.8 & 4.2 & -1 & 0 \\
\hline $10: 1 \mathrm{HF}$ & $w$ & 250 & 1100 & $\mathrm{~s}$ & $\mathrm{~s}$ & s & $\mathrm{s}$ & $\mathrm{s}$ & $\mathrm{s}$ & - \\
\hline $5: 1 \mathrm{BHF}$ & 11 & 140 & w & $<2$ & 0 & $\mathrm{~s}$ & $\begin{array}{ll}R & 0\end{array}$ & 0 & $<0.3$ & $P$ \\
\hline Pad Etch 4 & 1.9 & $R<15$ & $<2$ & s. & $\mathrm{s}$ & s & $\mathrm{s}$ & $\mathrm{s}$ & $\mathrm{s}$ & : \\
\hline Phosphoric & $>500$ & 980 & - &. & 0 & - & 0 & 100 & - & - \\
\hline Al Etch A & 530 & 660 & 0 &. &. & - & -1 & $\begin{array}{ll}T & 0 \\
\end{array}$ &. & 1.0 \\
\hline Ti Etch & 150 & 240 & 1100 &. & - & - &. & 0 & $\mathrm{~s}$ & - \\
\hline CR-7 & 3.8 & $\mathrm{~s}$ & $<2$ & 60 & $\begin{array}{ll}R & 0 \\
\end{array}$ & $\mathrm{~s}$ & $<0.7$ & 170 & 150 & 110 \\
\hline CR-14 & 0 & 0.8 & $<2$ & 15 & - & - &. & 93 & w & 120 \\
\hline Moly Etch & $>20$ &. &. &. & - &. &. & $\begin{array}{ll}R & 0\end{array}$ & - & - \\
\hline $\mathrm{H}_{2} \mathrm{O}_{2} \quad 50^{\circ} \mathrm{C}$ & $\begin{array}{ll}T & 0 \\
\end{array}$ & 0.25 & - & - & - & - &. & 110 & $w$ & - \\
\hline $\mathrm{Cu} \mathrm{FeCl}{ }_{3} 200$ & 35 & $w$ & - &. & - & - & -1 & 0.053 & $\mathrm{~s}$ & - \\
\hline Cu APS 100 & $<0.3$ & - &. &. & - &. &. & 0 & $\mathrm{~s}$ & - \\
\hline Dil. Aqua regia & 600 & $w$ & $<0.5$ &. & 0 & s) & $<2$ & 0 & s & - \\
\hline$A U-5$ & & - & - &. & - & - & - & 0 & S & - \\
\hline $\mathrm{NiCr}$ TFN & $>46$ &. & - & -1 & - & -1 & & $>170$ & $w$ & $w$ \\
\hline Phos+Sulf & $w$ & $w$ &. &. & - &. & & $1>500$ & & \\
\hline Piranha & $>5200$ & $w$ & 240 & - & 6.3 & $\mathrm{~s}$ & $\begin{array}{ll}T & 0\end{array}$ & $>16$ & 5.7 & $R \quad 0$ \\
\hline Microstrip & - & - &. & - & - & - & - & - & $\cdot$ & - \\
\hline Acetone & $\mathrm{s}$ & 0 & 0 & s) & $\mathrm{s}$ & s) & $\mathrm{s}$ & s) & $\mathrm{s}$ & S \\
\hline Methanol & $\mathrm{s}$ & s & $\mathrm{s}$ & $\mathrm{s}$ & $\mathrm{s}$ & $\mathrm{s}$ & s. & $\mathrm{s}$ & s. & S \\
\hline IPA & s) & s & s & s) & $\mathrm{s}$ & s & s & s & S & S \\
\hline $\mathrm{XeF}_{2}$ & s & 0 & 29 & $w$ & $w$ & $w$ & $w$ & - & - & - \\
\hline HF vapor & $\mathrm{R}$ & $\mathrm{R}$ & $\mathrm{R}$ & - & - & - & -1 & s & s & - \\
\hline Technics $\mathrm{O}_{2}$ & $\mathrm{~s}$ & 0 & 0 & $\mathrm{~s}$ & s & s & s) & $\mathrm{s}$ & s & $S$ \\
\hline DRIE HF mech. &. & - & 4.9 & - & - & - & - & - & & - \\
\hline DRIE HF ES &. & - & - & - & - & - &. &. & - & - \\
\hline DRIE LF mech. & - & -1 & - &. & - & - &. & - & -1 & - \\
\hline DRIE LF ES & - & - & - &. & - & - & - & - & -1 & - \\
\hline STS $320 \mathrm{SF}_{6}$ & - & -1 & - &. & w & $w$ & $w$ & $<1$ & $<0.7$ & S \\
\hline STS $\mathrm{SF}_{6}+\mathrm{O}_{2}$ & $<2.8$ & - & - & - & 26 & $w$ & 37 & $<1$ & $<0.9$ & S \\
\hline STS $320 \mathrm{CF}_{4}$ & $\mathrm{~s}$ & $\mathrm{~s}$ & $\cdot$ & - & - & - & -1 & $<1$ & $<3$ & - \\
\hline $\mathrm{STS} \mathrm{CF}_{4}+\mathrm{O}_{2}$ & 0.87 & 1.5 &. & - & 14 & -1 & 21 & $<1.3$ & $<1.2$ & - \\
\hline Ion Mill & 73 & $w$ & 38 & $w$ & $w$ & 42 & 42 & 58 & 58 & W \\
\hline \multicolumn{11}{|c|}{$\begin{array}{l}\text { Evap = evaporated } \\
\text { Ion-Mill }=\text { Ion-mill deposited }\end{array}$} \\
\hline
\end{tabular}

chemical-vapor-deposition (LPCVD) furnace with the recipe $\mathrm{SiH}_{4}=74 \mathrm{sccm}, \mathrm{O}_{2}=105 \mathrm{sccm}, \mathrm{p}=500$ mtorr, $\mathrm{T}=495^{\circ} \mathrm{C}$. $\mathrm{RI}=1.46$, then annealed in $\mathrm{N}_{2}$ at $1000^{\circ} \mathrm{C}$ for $60 \mathrm{~min}$. LTO is used instead of thermal oxide in many applications due to its relatively low deposition temperature and the ability to deposit thick films on surfaces other than silicon. LTO should be annealed prior to the deposition of another film at higher temperature (e.g., polysilicon) because it will outgas hydrogen, which can cause the overlying film to bubble.

Unan. LTO LPCVD Tylan: LTO deposited in a Tylan LPCVD furnace with the recipe $\mathrm{SiH}_{4}=60 \mathrm{sccm}, \mathrm{O}_{2}=$ 90 sccm, $\mathrm{p}=300$ mtorr, $\mathrm{T}=450^{\circ} \mathrm{C}$, no anneal.

Ann. LTO LPCVD Tylan: LTO deposited as above, then annealed in $\mathrm{N}_{2}$ at $1000{ }^{\circ} \mathrm{C}$ for $60 \mathrm{~min} . \mathrm{RI}=1.46$. 
TABLE VI

Etch Rates of Mo, W, Ni, Pd, Pt, Cu, Ag, Au, TiW, NiCr, AND TiN (nm/min)

\begin{tabular}{|c|c|c|c|c|c|c|c|c|c|c|c|}
\hline Etch & $\begin{array}{c}\text { Molybdenum } \\
\text { Evap }\end{array}$ & $\begin{array}{l}\text { Tungsten } \\
\text { Sputtered }\end{array}$ & $\begin{array}{c}\text { Nickel } \\
\text { Evap } \\
\end{array}$ & $\begin{array}{c}\text { Palladium } \\
\text { Evap } \\
\end{array}$ & $\begin{array}{c}\text { Platinum } \\
\text { Evap } \\
\end{array}$ & $\begin{array}{c}\text { Copper } \\
\text { Evap } \\
\end{array}$ & $\begin{array}{l}\text { Silver } \\
\text { Evap }\end{array}$ & $\begin{array}{l}\text { Gold } \\
\text { Evap }\end{array}$ & $\begin{array}{c}10 T / / 90 \mathrm{~W} \\
\text { Ion-Mill }\end{array}$ & $\begin{array}{c}80 \mathrm{Ni} / 20 \mathrm{Cr} \\
\text { Evap }\end{array}$ & $\begin{array}{c}\text { TiN } \\
\text { Sputtered } \\
\end{array}$ \\
\hline Si Iso Etch & 11,000 & 13 & 21 & 0 & 0 & 37 & 49 & 0 & 23 & & soft \\
\hline $\mathrm{KOH}$ & 0 & 0 & 0 & 0 & 0 & $\begin{array}{ll}1 & 0\end{array}$ & $\begin{array}{ll}1 & 0\end{array}$ & 0 & $>300$ & & \\
\hline $10: 1 \mathrm{HF}$ & $\mathrm{s}$ & 0 & $\mathrm{~s}$ & $\mathrm{~s}$ & s & s & $\mathrm{s}$ & s & $\mathrm{s}$ & $s$ & s \\
\hline $5: 1 \mathrm{BHF}$ & $<0.3$ & $<2$ & $<1.1$ & 0 & 0 & $R<5$ & $R<5$ & 0 & $R<0.2$ & $R<1.5$ & 2.5 \\
\hline Pad Etch 4 & s & s & - & - & & & & -1 & & & \\
\hline Phosphoric & - & . &. &. & & 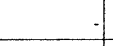 & & 0 & 2.5 & & \\
\hline Al Etch A & & & 29 & - & & $>2900$ & & & & & \\
\hline Ti Etch & - & 11 & -1 & - & -1 & - & 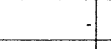 & - & 0 & & \\
\hline CR-7 & 3.3 & 3.2 & 1.7 & 0 & 0 & 280 & 450 & 0 & 0.62 & 11.2 & \\
\hline CR-14 & soft & 0 & $<2$ & 0 & $<3$ & 19 & & & - & 0.22 & $<2$ \\
\hline Moly Etch & 690 & 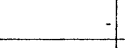 & -1 & - & 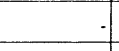 & & & - & 0 & & \\
\hline $\mathrm{H}_{2} \mathrm{O}_{2} \quad 50^{\circ} \mathrm{C}$ & - & 150 & - & - & - &. & &. & w & & \\
\hline $\mathrm{Cu} \mathrm{FeCl}{ }_{3} 200$ & & -1 & 21 & - & - & 3900 & $\begin{array}{ll}T & 0\end{array}$ & & & & \\
\hline Cu APS 100 & - & -1 & 0 & - & - & 2500 &. & - &. & & \\
\hline Dil. Aqua regia & 650 & 5.2 & 100 & 390 & 3.6 & 600 & w & 680 & 3.7 & & \\
\hline AU-5 & -1 & & 0 & \begin{tabular}{ll|} 
& 0 \\
\end{tabular} & $<2$ & $\begin{array}{ll}T & 0 \\
\end{array}$ & $\begin{array}{ll}T & 0 \\
\end{array}$ & 660 & & -1 & \\
\hline NiCr TFN & 680 & -1 & 13 & & & 690 & - &. & . & 83 & \\
\hline \multicolumn{12}{|l|}{ Phos+Sulf } \\
\hline Piranha & 18 & -1 & 380 & 3.0 & $<3$ & 88 & 600 & 0 & 0.78 & 92 & \\
\hline \multicolumn{12}{|l|}{ Microstrip } \\
\hline Acetone & $\mathrm{s}$ & s & $\mathrm{s}$ & $s$ & s & s & s & s & s & s & $S$ \\
\hline Methanol & s & s & s & $\mathrm{s}$ & s & $\mathrm{s}$ & s & s & s & s & s \\
\hline IPA & s & s & s & $\mathrm{s}$ & s & $\mathrm{s}$ & $\mathrm{s}$ & $\mathrm{s}$ & s & s & $\mathrm{s}$ \\
\hline $\mathrm{XeF}_{2}$ & $w$ & 80 & -1 & - & - & -1 & - & - & $w$ & - & - \\
\hline HF vapor & - & 0 & s &. & s & $\mathrm{R}$ & 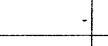 & s &. & -1 & \\
\hline Technics $\mathrm{O}_{2}$ & s & 0 & s & s & s & s & s & s & s & $\mathrm{s}$ & $S$ \\
\hline DRIE HF mech. & 5.7 & 4.9 & s & s & s & s & s & s & 4.6 & s & \\
\hline DRIE HF ES & - & - & - &. & - & - & -1 & - &. & - & - \\
\hline \multicolumn{12}{|l|}{ DRIE LF mech. } \\
\hline \multicolumn{12}{|l|}{ DRIE LFES } \\
\hline STS $320 \mathrm{SF}_{6}$ & 73 & $w$ & s & s & s & s & 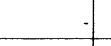 & s & $w$ & s & \\
\hline STS SF ${ }_{6}+\mathrm{O}_{2}$ & 130 & w & 0.71 & 3.1 & 7.4 & $\mathrm{~s}$ &. & $\mathrm{~s}$ & 550 & 3.7 & \\
\hline STS $320 \mathrm{CF}_{4}$ & $w$ & 7 & -1 & -1 & - & -1 & 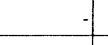 & s & - & -1 & \\
\hline STS $\mathrm{CF}_{4}+\mathrm{O}_{2}$ & 150 &. & -1 & 1.0 & 1.4 & - & - & 8.3 & 49 & 0 & - \\
\hline Ion Mill & 54 & 38 & 66 & 130 & 88 & 110 & 220 & 170 & $w$ & 18 & $w$ \\
\hline $\begin{array}{l}\text { Notation: } \\
W=\text { Etch known } \\
\text { F = Etch known } \\
S=\text { Etch rate knd } \\
\text { R = Film was vis } \\
T=\text { Thicker after } \\
P=\text { Some of film } \\
I=\text { Incubation tim } \\
C=\text { Film congeal } \\
\text { soft = a soft mate }\end{array}$ & $\begin{array}{l}\text { to work, but etc } \\
\text { o be fast, but et } \\
\text { own to be slow } \\
\text { bly roughened } \\
\text { etch (due to sw } \\
\text { peeled during } \\
\text { ee before etchin } \\
\text { ed } \\
\text { erial remained a }\end{array}$ & $\begin{array}{l}\text { ch rate not me } \\
\text { tch rate not } n \\
\text { or zero, but } \\
\text { or attacked } \\
\text { velling or com } \\
\text { etch or when } \\
\text { ig fully starts } \\
\text { after etching }\end{array}$ & $\begin{array}{l}\text { asured } \\
\text { easured } \\
\text { ch rate not } m \\
\text { ound format } \\
\text { nsed }\end{array}$ & $\begin{array}{l}\text { neasured } \\
\text { tion) }\end{array}$ & $\begin{array}{l}\text { Evap }=\text { eva } \\
\text { Ion-Mill }=10\end{array}$ & $\begin{array}{l}\text { orated } \\
\text { i-mill deposit }\end{array}$ & & & & & \\
\hline
\end{tabular}

Unan. PSG LPCVD Tylan: Phosphosilicate glass (PSG) deposited in a Tylan LPCVD furnace with the recipe $\mathrm{SiH}_{4}=$ $60 \mathrm{sccm}, \mathrm{O}_{2}=90 \mathrm{sccm}, \mathrm{PH}_{3}=10.3 \mathrm{sccm}, \mathrm{p}=300 \mathrm{mtorr}$, $\mathrm{T}=450{ }^{\circ} \mathrm{C}$, no anneal. $\mathrm{RI}=1.47$. PSG is LTO doped with $\mathrm{P}_{2} \mathrm{O}_{5}$, typically in the 5-10\% range. It is used as a sacrificial layer in micromachining because it etches much faster than undoped LTO in HF solutions.

Ann. PSG LPCVD Tylan: PSG deposited as above, then annealed in $\mathrm{N}_{2}$ at $1000^{\circ} \mathrm{C}$ for $60 \mathrm{~min}$. $\mathrm{RI}=1.48$. Like pure $\mathrm{LTO}$, PSG should be annealed prior to the deposition of another film at high temperature (e.g., polysilicon) because it will outgas hydrogen, which can cause the overlying film to bubble. Annealing results in a lower etch rate in HF solutions.

Oxide PECVD Unannealed: Silicon dioxide deposited in an STS 310 plasma-enhanced chemical-vapor-deposition (PECVD) system with the recipe $2 \% \mathrm{SiH}_{4} / \mathrm{Ar}=500 \mathrm{sccm}$, $\mathrm{N}_{2} \mathrm{O}=1410 \mathrm{sccm}, \mathrm{P}=10 \mathrm{~W}$ at $13.56 \mathrm{MHz}, \mathrm{p}=800 \mathrm{mtorr}$, $\mathrm{T}=300{ }^{\circ} \mathrm{C}$. RI $=1.46$. PECVD silicon dioxide usually contains 2-9 atomic \% hydrogen [5]. PECVD oxide can be deposited at an even lower temperature than LPCVD films. 
TABLE VII

Etch RATES OF RESISTS, PARYLENE, AND POLYIMIDE (nm/min)

\begin{tabular}{|c|c|c|c|c|c|c|c|}
\hline Etch & $\begin{array}{c}\text { S1822 } \\
\text { Positive } \\
\text { Photoresist } \\
\end{array}$ & $\begin{array}{c}\text { OCG } 820 \\
\text { Positive } \\
\text { Photoresist } \\
\end{array}$ & $\begin{array}{c}\text { Futurrex } \\
\text { Positive } \\
\text { Photoresist } \\
\end{array}$ & $\begin{array}{c}\text { Futurrex } \\
\text { Negative } \\
\text { Photoresist } \\
\end{array}$ & $\begin{array}{c}\text { Act. Mrk. } \\
\text { Resist } \\
\text { Pen }\end{array}$ & $\begin{array}{c}\text { Parylene } \\
\text { Type C } \\
\end{array}$ & $\begin{array}{l}\text { Spin-On } \\
\text { PI } 2556 \\
\text { Polymide } \\
\end{array}$ \\
\hline Si Iso Etch & P. $\quad 0$ & 0 & P $\quad 21$ & 290 & - & 0.019 & 0 \\
\hline $\mathrm{KOH}$ & $>17,900$ & $\mathrm{~F}$ & $>13,000$ & $>18,000$ & $\mathrm{~F}$ & 0.42 & $T$ \\
\hline $10: 1 \mathrm{HF}$ & $s$ & 0 & - & - & $\mathrm{s}$ & 4.4 & $S$ \\
\hline 5:1 BHF & 0 & 0 & 21 & 5.0 & $<3$ & 0.16 & 5.5 \\
\hline Pad Etch 4 & $\begin{array}{ll}T & 0\end{array}$ & - & - & - & - &. & - \\
\hline Phosphoric & P 120 & 55 & $\begin{array}{ll}P & 77 \\
\end{array}$ & $>400$ & - & 0.55 & $\therefore$ \\
\hline Al Etch A & 0 & 0 & - & - & - &. & - \\
\hline Ti Etch & $<0.5$ & 0 & - & - & - & - & - \\
\hline CR-7 & 0 & $\mathrm{~s}$ & 0 & $<0.5$ & 3.8 & - & 0 \\
\hline CR-14 & 0.24 & $\mathrm{~s}$ & $\mathrm{~s}$ & $\mathrm{~s}$ & $\mathrm{~s}$ & - & S \\
\hline Moly Etch & 230 & - & - & - & - &. & - \\
\hline $\mathrm{H}_{2} \mathrm{O}_{2} \quad 50^{\circ} \mathrm{C}$ & $\begin{array}{ll}R T & 0 \\
\end{array}$ & $\mathrm{~s}$ & $\mathrm{~s}$ & $\mathrm{~s}$ & s & $\begin{array}{ll}T & 0 \\
\end{array}$ & $\mathrm{~S}$ \\
\hline $\mathrm{Cu} \mathrm{FeCl}{ }_{3} 200$ & 0.48 & $\mathrm{~s}$ & $\mathrm{~s}$ & s & $\mathrm{s}$ & $\cdot$ & $S$ \\
\hline Cu APS 100 & 0 & $\mathrm{~s}$ & s & s & $\mathrm{s}$ & - & S \\
\hline Dil. Aqua regia & 0 & s & $\begin{array}{ll}\mathrm{T} & 0 \\
\end{array}$ & $\begin{array}{ll}T & 0\end{array}$ & $\begin{array}{ll}T & 0 \\
\end{array}$ & - & T 0 \\
\hline AU-5 & $\begin{array}{ll}P & 0 \\
\end{array}$ & S & $\mathrm{s}$ & s & $\mathrm{s}$ & - & $S$ \\
\hline NiCr TFN & 0.57 & $\mathrm{~s}$ & s & $\mathrm{s}$ & S & - & S \\
\hline Phos+Sulf & $\mathrm{F}$ & $\mathrm{F}$ & $\mathrm{F}$ & $\mathrm{F}$ & $\mathrm{F}$ & - & - \\
\hline Piranha & $>92,000$ & $\mathrm{~F}$ & $>59,000$ & $>59,000$ & $>15,000$ & 2.6 & $>17,000$ \\
\hline Microstrip & $>94,000$ & $\mathrm{~F}$ & $>85,000$ & $>60,000$ & $>11,000$ & $\cdot$ & 520 \\
\hline Acetone & $>176,000$ & $\mathrm{~F}$ & $>120,000$ & $>87,000$ & $>26,000$ & 0.77 & 0 \\
\hline Methanol & $>36,000$ & .1 & $P C>16,000$ & $>27,000$ & $\mathrm{P}$ & 0.093 & 0 \\
\hline IPA & $>1000$ & - & 480 & 910 & -1 & $\begin{array}{ll}T & 0\end{array}$ & 0 \\
\hline $\mathrm{XeF}_{2}$ & $\mathrm{~s}$ & 0 & $\mathrm{~s}$ & $\mathrm{~s}$ & $\mathrm{~s}$ & - & S \\
\hline HF vapor & - & $\begin{array}{ll}P & 0 \\
\end{array}$ & $\mathrm{~s}$ & $\mathrm{~s}$ & $\mathrm{~s}$ & - & S \\
\hline Technics $\mathrm{O}_{2}$ & 300 & 340 & 200 & 470 & 370 & 220 & 370 \\
\hline DRIE HF mech. & 30 & $w$ & $w$ & $w$ & 54 & - & - \\
\hline DRIE HF ES & $w$ & $w$ & $w$ & $w$ & $w$ & 27 & - \\
\hline DRIE LF mech. & 35 & $w$ & 34 & $w$ & $w$ & - & - \\
\hline DRIE LFES & $w$ & $w$ & $w$ & $w$ & $w$ & 15 & - \\
\hline STS $320 \mathrm{SF}_{6}$ & 120 & $w$ & 164 & 140 & $w$ & - & 140 \\
\hline STS SF $6+\mathrm{O}_{2}$ & 180 &. & $1 \quad 130$ & 320 & 170 & - & 200 \\
\hline STS $320 \mathrm{CF}_{4}$ & 42 & $w$ & $w$ & 72 & $w$ & & - \\
\hline STS $\mathrm{CF}_{4}+\mathrm{O}_{2}$ & 130 &. & $1 \quad 100$ & 190 & 52 & - & 160 \\
\hline Ion Mill & $R<100$ & $w$ & $w$ & $w$ & $w$ & $w$ & $w$ \\
\hline \multicolumn{8}{|c|}{$\begin{array}{l}\text { Notation: } \\
W=\text { Etch known to work, but etch rate not measured } \\
F=\text { Etch known to be fast, but etch rate not measured } \\
S=\text { Etch rate known to be slow or zero, but etch rate not measured } \\
R=\text { Film was visibly roughened or attacked } \\
T=\text { Thicker after etch (due to swelling or compound formation) } \\
P=\text { Some of film peeled during etch or when rinsed } \\
I=\text { Incubation time before etching fully starts } \\
C=\text { Film congealed } \\
\text { soft = a soft material remained after etching }\end{array}$} \\
\hline
\end{tabular}

Oxide PECVD Annealed: Silicon dioxide deposited as above, then annealed in $\mathrm{N}_{2}$ at $1000{ }^{\circ} \mathrm{C}$ for $60 \mathrm{~min}$. $\mathrm{RI}=1.46$. Annealing densifies the film, resulting in a lower etch in HF solutions.

Oxide Ion-Milled: Silicon dioxide ion-mill-deposited from a silicon-dioxide target in a Commonwealth Scientific system with argon ions at $1250 \mathrm{~V}$, current density of about $2 \mathrm{~mA} / \mathrm{cm}^{2}$, $\mathrm{p}=3 \times 10^{-4}$ torr. $\mathrm{RI}=1.57$. Ion milling is the lowest temper- ature deposition method studied here, with the wafer remaining near room temperature.

\section{Silicon Nitride}

Stoich. Si Nit. LPCVD: Stoichiometric silicon nitride $\left(\mathrm{Si}_{3} \mathrm{~N}_{4}\right)$ deposited in a Tylan LPCVD furnace with the recipe $\mathrm{SiCl}_{2} \mathrm{H}_{2}=25 \mathrm{sccm}, \mathrm{NH}_{3}=75$ sccm, $\mathrm{p}=200$ mtorr, $\mathrm{T}=800{ }^{\circ} \mathrm{C}$. RI $=1.99$. Silicon nitride is used as an etch 
mask, thermal-oxidation mask, and wafer-capping material due to its low permeability to moisture. It typically has a high tensile residual stress.

Si-Rich Si Nit. LPCVD: Silicon-rich, low-stress silicon nitride deposited in a Calogic LPCVD furnace with the recipe $\mathrm{SiCl}_{2} \mathrm{H}_{2}=159 \mathrm{sccm}, \mathrm{NH}_{3}=40.5 \mathrm{sccm}, \mathrm{p}=280$ mtorr, $\mathrm{T}=850{ }^{\circ} \mathrm{C}$. RI $=2.24$. Low-stress silicon nitride typically has a tensile residual stress below $50 \mathrm{MPa}$, allowing its use in micromachining as a freestanding film.

PECVD Silicon Nit. Low RI: Silicon nitride deposited in an STS 310 PECVD system with a relatively low ratio of silicon to nitrogen with the recipe $2 \% \mathrm{SiH}_{4} / \mathrm{Ar}=100 \mathrm{sccm}, \mathrm{N}_{2}=2000$ sccm, power alternating between $\mathrm{P}=60 \mathrm{~W}$ at $13.56 \mathrm{MHz}$ for $6 \mathrm{~s}$ and $25 \mathrm{~W}$ at $187 \mathrm{kHz}$ for $3 \mathrm{~s}, \mathrm{p}=750$ mtorr, $\mathrm{T}=$ $350{ }^{\circ} \mathrm{C}$. RI $=1.88$. PECVD silicon nitride is deposited at a much lower temperature than LPCVD and can contain well over 10 atomic percent hydrogen [5], and is therefore frequently written as $\mathrm{Si}_{\mathrm{x}} \mathrm{N}_{\mathrm{y}} \mathrm{H}_{\mathrm{z}}$ or $\mathrm{SiN}: \mathrm{H}$. High frequency typically yields tensile silicon nitride films; low frequency typically gives compressive films. "Mixed-frequency" nitride such as this has a lower absolute value of stress.

PECVD Silicon Nit. High RI: Silicon nitride deposited as above, but with a relatively high ratio of silicon to nitrogen by increasing the flow of $2 \% \mathrm{SiH}_{4} / \mathrm{Ar}$ to $500 \mathrm{sccm}$. $\mathrm{RI}=2.53$. This PECVD silicon nitride has a significantly lower etch rate in HF solutions than its low-index counterpart above, but still etches much faster than LPCVD nitride.

\section{Aluminum Oxide}

Sapphire Wafer: Clear, colorless, pure crystalline aluminum oxide wafer. (Aluminum oxide is also known as alumina or corundum.) These wafers are referred to as sapphire in the electronics industry, although true sapphire has a small amount of titanium, giving it a blue color. Sapphire has been used as substrate for silicon-on-insulator (SOI) wafers. Sapphire was the most chemically inert material tested for this paper.

Aluminum Oxide Ion-Milled: Aluminum oxide ion-milldeposited in a Commonwealth Scientific system from a sapphire target with argon ions at $1250 \mathrm{~V}$, current density of about $2 \mathrm{~mA} / \mathrm{cm}^{2}$. The sapphire was held to a copper backing plate with copper-loaded vacuum grease. A small area of this grease was exposed during deposition. Adhesion of the film to the wafer was good. Like silicon dioxide, aluminum oxide is relatively inert. Aluminum oxide films have been used as dielectrics and protective coatings.

Aluminum Oxide Evaporated: Aluminum oxide electronbeam evaporated from an alumina source. Wafer temperature was not controlled and was near room temperature during deposition. $\mathrm{RI}=1.66$.

\section{E. Aluminum}

Aluminum Evaporated: Aluminum evaporated from a tungsten wire in a Veeco evaporator. Aluminum is commonly used for electrical interconnections due to its ease of use and good conductivity. It is well known to have good adhesion to silicon and silicon dioxide.

$A l+2 \%$ Si Sputtered: Aluminum sputtered using argon in a CPA 9900 sputtering system with the recipe $\mathrm{p}=6$ mtorr,
$\mathrm{P}=4.5 \mathrm{~kW}$, track speed $=10 \mathrm{~cm} / \mathrm{min}$. Aluminum with a small percentage of silicon, added to prevent "spiking" of metal into the substrate, is a common material for IC interconnects.

\section{F. Transition Metals}

Titanium Sputtered: Titanium sputtered using argon in a CPA 9900 sputtering system with the recipe $p=6$ mtorr, $\mathrm{P}=4.5 \mathrm{~kW}$, track speed $=10 \mathrm{~cm} / \mathrm{min}$. Titanium is well known to have good adhesion to silicon and silicon dioxide, and is frequently used as an adhesion layer for less-adhesive metals such as gold.

Vanadium Evaporated: Vanadium electron-beam evaporated in a CHA evaporator at a rate of $0.1 \mathrm{~nm} / \mathrm{s}$. The pressure was below $9 \times 10^{-7}$ torr at the start of the evaporation. Vanadium was found to have good adhesion to silicon and silicon dioxide, as evaluated by firmly pressing $3 \mathrm{M}$ Scotch Tape onto the film, then peeling the tape off without removing the metal. Vanadium thus has potential as an alternative adhesion layer.

Niobium Ion Milled: Niobium ion-mill-deposited in a Commonwealth Scientific system with argon ions at $1250 \mathrm{~V}$, current density of about $2 \mathrm{~mA} / \mathrm{cm}^{2}, \mathrm{p}=3 \times 10^{-4}$ torr. Niobium was found to have good adhesion to silicon, so it has potential as an alternative adhesion layer.

Tantalum Evaporated: Tantalum electron-beam evaporated in a CHA evaporator at a rate of $0.1 \mathrm{~nm} / \mathrm{s}$. The pressure was below $9 \times 10^{-7}$ torr at the start of the evaporation. Tantalum was found to have good adhesion to silicon and silicon dioxide, so it has potential as an alternative adhesion layer. As one of the high-melting-temperature metals, tantalum has been used as a hot filament in inert atmospheres.

Tantalum Ion-Milled: Tantalum ion-mill-deposited in a Commonwealth Scientific system with argon ions at $1250 \mathrm{~V}$, current density of about $2 \mathrm{~mA} / \mathrm{cm}^{2}, \mathrm{p}=3 \times 10^{-4}$ torr. Tantalum deposited using this method also was found to have good adhesion to silicon and silicon dioxide.

Chromium Evaporated: Chromium evaporated from a tungsten strip in a CHA evaporator at a rate of $0.1 \mathrm{~nm} / \mathrm{s}$. The pressure was below $9 \times 10^{-7}$ torr at the start of the evaporation. Chromium is well known to have good adhesion to silicon and silicon dioxide, and is frequently used as an adhesion layer for less-adhesive metals such as gold.

Chromium Ion Milled: Chromium ion-mill-deposited in a Commonwealth Scientific system with argon ions at $1250 \mathrm{~V}$, current density of about $2 \mathrm{~mA} / \mathrm{cm}^{2}, \mathrm{p}=3 \times 10^{-4}$ torr.

Patterned Cr on Au Evaporated: Evaporated chromium previously lithographically patterned and etched, sitting on a continuous layer of gold (which itself has a chromium adhesion layer). This was done to study the potential electrochemical effect of this commonly used system of two metals in contact.

Moly Evaporated: Molybdenum electron-beam evaporated in a CHA evaporator at a rate of $0.5 \mathrm{~nm} / \mathrm{s}$. The pressure was below $9 \times 10^{-7}$ torr at the start of the evaporation. The evaporated molybdenum used in these tests had good adhesion to silicon, but was found to have only fair adhesion to oxide: it did not peel when Scotch tape was lightly pressed, but did when firmly pressed and removed. We have found it to have good adhesion when sputtered. Molybdenum has a relatively low thermal coefficient of expansion and has been used as a heat sink attached 
to silicon. It has been used in MEMS as the electrode material for film-bulk acoustic resonators [6].

Tungsten Sputtered: Tungsten sputtered using argon in a CPA 9900 sputtering system with the recipe $\mathrm{p}=6$ mtorr, $\mathrm{P}=4.5 \mathrm{~kW}$, track speed $=10 \mathrm{~cm} / \mathrm{min}$. An adhesion layer of titanium was used under the tungsten. Tungsten also has a relatively low thermal coefficient of expansion and has been used as a heat sink attached to silicon. Tungsten has been used in MEMS as a hot filament in vacuum [7] and as an interconnect material compatible with high-temperature processing.

Nickel Evaporated: Nickel electron-beam evaporated in a $\mathrm{CHA}$ evaporator at a rate of $0.3 \mathrm{~nm} / \mathrm{s}$. The pressure was below $9 \times 10^{-7}$ torr at the start of the evaporation. A 10 -nm adhesion layer of chromium was used under the nickel. Nickel has been used as a diffusion-barrier layer in contacts, a seed layer for electroplating (immediately after removing its native oxide), and as the metal to which solder adheres.

Palladium Evaporated: Palladium electron-beam evaporated in a CHA evaporator at a rate of $0.15 \mathrm{~nm} / \mathrm{s}$. The pressure was below $9 \times 10^{-7}$ torr at the start of the evaporation. A $10-\mathrm{nm}$ adhesion layer of chromium was used under the palladium. Like platinum, palladium has been used as catalyst, for example in the hydrogenation of carbon compounds [2]. In these tests, it was etched more easily than platinum.

Platinum Evaporated: Platinum electron-beam evaporated in a CHA evaporator at a rate of $0.15 \mathrm{~nm} / \mathrm{s}$. The pressure was below $9 \times 10^{-7}$ torr at the start of the evaporation. A $10-\mathrm{nm}$ adhesion layer of chromium was used under the platinum. Platinum has been used in thin-film heaters, resistive temperature sensors, and as a diffusion-barrier layer in contacts due to its inertness and stability. It is also well-known as a catalyst and has been used in fine-grain form (for large surface area) in microscale fuel cells. Platinum is often used as the electrode layer on which lead zirconate titanate (PZT) is grown for good crystal orientation. In this work it was the most difficult metal to etch.

Copper Evaporated: Copper evaporated from a tungsten boat in a CHA evaporator at a rate of $0.17 \mathrm{~nm} / \mathrm{s}$. The pressure was below $9 \times 10^{-7}$ torr at the start of the evaporation. A $10-n m$ adhesion layer of chromium was used under the copper. Copper is second to only to silver in its electrical conductivity, and is presently used in high-speed integrated circuits. Plated copper has been employed in MEMS for high-Q inductors.

Silver Evaporated: Silver electron-beam evaporated in a CHA evaporator at a rate of $0.5 \mathrm{~nm} / \mathrm{s}$. The pressure was below $9 \times 10^{-7}$ torr at the start of the evaporation. A $10-\mathrm{nm}$ adhesion layer of chromium was used under the silver. Silver was found to form a dark tarnish (due to sulfide formation, not oxidation [8]) within days of deposition.

Gold Evaporated: Gold electron-beam evaporated in a CHA evaporator at a rate of $0.5 \mathrm{~nm} / \mathrm{s}$. The pressure was below $9 \times$ $10^{-7}$ torr at the start of the evaporation. A $10-\mathrm{nm}$ adhesion layer of chromium was used under the gold. Gold, which is an excellent electrical conductor, has many applications in electronics: Because it does not corrode like copper and aluminum, it has been used in mechanical contacts and bond pads. Micromachined switches have employed gold or its alloys. Gold surfaces are wetted well by many solders, but are also rapidly dissolved, so an underlying metal layer (e.g., Ni or Pt) must be used.

\section{G. Metal Alloys and Compound}

$10 \mathrm{Ti} / 90 \mathrm{~W}$ Ion-Milled: $10 \%$ titanium/90\% tungsten alloy ion-mill-deposited in a Commonwealth Scientific system with argon ions at $1250 \mathrm{~V}$, current density of about $2 \mathrm{~mA} / \mathrm{cm}^{2}, \mathrm{p}=$ $3 \times 10^{-4}$ torr. This alloy is commonly used as a combination adhesion/diffusion-barrier layer.

$80 \mathrm{Ni} / 20 \mathrm{Cr}$ Evaporated: $80 \%$ nickel $/ 20 \%$ chromium (at the source) alloy evaporated from a tungsten wire in a Veeco evaporator. Due to their inertness at higher temperature and relatively low thermal conductivity, nickel-chromium alloys have been used for heaters.

TiN Sputtered: Titanium nitride sputtered using in a Varian/Novellus MI2 sputtering system with the recipe argon = $55 \mathrm{sccm}$, nitrogen $=65 \mathrm{sccm}, \mathrm{P}=7.2 \mathrm{~kW}, \mathrm{~T}=375^{\circ} \mathrm{C}$. TiN is used as an adhesion layer.

\section{H. Resists}

S1822 Positive Photoresist: Shipley Microposit S1822 positive photoresist, dispensed, and spun at $4600 \mathrm{rpm}$ for $30 \mathrm{~s}$ for a thickness of $2.2 \mu \mathrm{m}$, soft baked on a hot plate at $115^{\circ} \mathrm{C}$ for $60 \mathrm{~s}$, then hard baked in an oven at $110^{\circ} \mathrm{C}$ for $30 \mathrm{~min} \mathrm{RI}=1.65$.

OCG Positive 820 Photoresist: OCG (formerly KTI) 820 photoresist, dispensed, and spun at $6000 \mathrm{rpm}$ for $30 \mathrm{~s}$ for a thickness of $1.1 \mu \mathrm{m}$, soft baked on a hot plate, then hard baked in an oven at $120^{\circ} \mathrm{C}$ for $30 \mathrm{~min} \mathrm{RI}=1.65$.

Futurrex Positive Photoresist: Futurrex PR1-4000A photoresist, dispensed, and spun at $2000 \mathrm{rpm}$ for $45 \mathrm{~s}$ for a thickness of $4 \mu \mathrm{m}$, and soft baked at $120{ }^{\circ} \mathrm{C}$ for $90 \mathrm{~s}$ on a hot plate (no hard bake was done per the manufacturer's instructions). $\mathrm{RI}=1.65$. This photoresist is formulated for a slow plasma-etch rate. $\mathrm{In}_{\mathrm{SF}_{6}}$ and $\mathrm{CF}_{4}$-based plasmas, it was found to barely etch for about $10 \mathrm{~min}$ (an "incubation time"), after which it started to etch more rapidly.

Futurrex Negative Photoresist: Futurrex NR5-8000 photoresist, dispensed, and spun at $5000 \mathrm{rpm}$ for $45 \mathrm{~s}$ for a thickness of $6 \mu \mathrm{m}$, and soft baked at $145^{\circ} \mathrm{C}$ for $60 \mathrm{~s}$ on a hot plate (no hard bake was done per the manufacturer's instructions). Unlike most photoresists, Futurrex positive and negative photoresists use the same developer.

Action Marker Resist Pen: ITW Dymon Action Marker Fine Line 33 PC-901 Blue resist pen, written onto a wafer and allowed to dry (no bake). This resist pen is convenient for rapidly masking an area or touching up photoresist before a wet or dry etch. It is rapidly stripped in acetone or photoresist stripper. Thicknesses were measured using profilometry.

\section{Parylene and Polyimide}

Parylene Type C: Parylene Type $\mathrm{C}$ deposited using a Specialty Coating Systems Model PDS 2010 Labcoter 2 system by heating the solid source material, the dimer di-para-xylylene, to $175^{\circ} \mathrm{C}$ to sublimate it, then heating the dimer vapor to $690^{\circ} \mathrm{C}$ to cleave the molecules into the monomer para-xylylene, finally depositing it on the substrate at room temperature and $\mathrm{p}=25$ mtorr to form a film of the polymer poly-(para-xylylene). Parylene is the trade name of the polymer, given by Union Carbide. $\mathrm{RI}=1.65$. 
There are several types of Parylene: Parylene-N can be envisioned as a polymer of benzene rings with $\mathrm{CH}_{2}$ groups on opposite ends. Parylene- $\mathrm{C}$ has a chlorine atom substituted for one of the hydrogen atoms in the benzene rings, while Parylene-D has two such substitutions. Parylenes are very inert chemically: of the etches tested, Parylene-C only had a significant etch rate in the oxygen plasma and ion mill (it also etched in a separate test in an $\mathrm{SF}_{6}$ plasma). Deposition is conformal and pinhole-free. Parylenes are also biocompatible and have a relatively low gas permeability. The flexibility of Parylene films has allowed their use in such MEMS applications as miniature valves and wings [9].

Spin-On PI 2556 Polyimide: DuPont PI2556 spin-on polyimide, dispensed, and spun at $4000 \mathrm{rpm}$ for $60 \mathrm{~s}$ for a thickness of $1.1 \mu \mathrm{m}$, held at $20{ }^{\circ} \mathrm{C}$ for $30 \mathrm{~min}$, then baked at $100{ }^{\circ} \mathrm{C}$ for $30 \mathrm{~min}$, and finally at $250{ }^{\circ} \mathrm{C}$ for $120 \mathrm{~min}$ (the manufacturer's instructions call for the last bake to be $300{ }^{\circ} \mathrm{C}$ for complete crosslinking). $\mathrm{RI}=1.82$.

\section{ETCH DESCRIPTIONS AND INFORMATION}

The wet and dry etches are described below, listed by the abbreviation labels (in italics) in the etch-rate tables. Information about the etch properties is included. Further documentation, including many chemical reactions, was presented previously [3].

\section{A. Wet Etches}

Si Iso Etch: Isotropic Silicon Etchant "Trilogy Etch," mixed from 126 parts $\mathrm{HNO}_{3}$ : 60 parts $\mathrm{H}_{2} \mathrm{O}: 5$ parts $\mathrm{NH}_{4} \mathrm{~F}$ ). $\mathrm{T} \approx$ $20{ }^{\circ} \mathrm{C}$ (room temperature). This solution should be mixed several hours before use to yield a stable etch rate. Similar commercial preparations are also available. During use, agitation increases uniformity. The solution gets weaker with use. Silicon etching in hydrofluoric acid-nitric acid-water (HF-nitric) and hydrofluoric acid-nitric acid-acetic acid (HNA) etch systems has been well characterized by Schwartz and Robbins [10].

This etchant is intended for isotropically etching silicon, both polycrystalline and single-crystal. It can be used to round off sharp corners formed during orientation-dependent etching and scallops and ridges formed during deep-reactive ion etching (DRIE) to lower the stress concentration of mechanical components. Silicon iso etch was also found to etch graphite, vanadium, niobium, and molybdenum at moderate to very high rates. Due to the small amount of HF formed upon mixing, it also slowly etches silicon dioxide. Photoresist can be normally be used as a mask with this etchant, although two of the resists used in these tests peeled.

Silicon and many metals are etched by the same sequence of steps in HNA etchants: Nitric acid is a powerful oxidizing agent. In nitric acid alone, the silicon or metal is oxidized to form a tough oxide film. The addition of hydrofluoric acid to the solution causes some of these metal oxides to be etched. A representative overall reaction is that for niobium [11]:

$$
\begin{aligned}
\mathrm{Nb}+5 \mathrm{HNO}_{3(\mathrm{aq})}+6 \mathrm{HF}_{(\mathrm{aq})} & \rightarrow \mathrm{H}^{+} \\
+ & \mathrm{NbF}_{6}^{-}+5 \mathrm{NO}_{2(\mathrm{~g})}+6 \mathrm{H}_{2} \mathrm{O}_{(\mathrm{l})} .
\end{aligned}
$$

$\mathrm{KOH}: 30 \%$ potassium hydroxide (by weight) in water. $\mathrm{T}=$ $80^{\circ} \mathrm{C}$. This solution can be mixed from solid pellets and water,
TABLE VIII

ETCH RATES OF THERMAL OXIDE IN VARIOUS DILUTIONS OF HF AND $\mathrm{BHF}(\mathrm{nm} / \mathrm{min})$

\begin{tabular}{l|r}
\hline \multicolumn{1}{c|}{ Etchant } & \multicolumn{1}{|c}{$\begin{array}{c}\text { Thermal } \\
\text { Oxide } \\
\text { Etch Rate }\end{array}$} \\
\hline Conc. HF (49\%) & 2300 \\
\hline $10: 1 \mathrm{HF}$ & 23 \\
\hline $25: 1 \mathrm{HF}$ & 9.7 \\
\hline $100: 1 \mathrm{HF}$ & 2.3 \\
\hline & 100 \\
\hline $5: 1 \mathrm{BHF}$ & 50 \\
\hline $10: 1 \mathrm{BHF}$ & \\
\hline $\begin{array}{l}\text { Notation: } \\
\text { BHF }=\text { buffered hydrofluoric acid } \\
\text { conc. }=\text { concentrated }\end{array}$ &
\end{tabular}

or from a diluted commercially prepared aqueous solution. It is used for crystal-orientation-dependent etching (ODE) of silicon, for etching n-type silicon to a heavily-doped p-type etch stop, and for electrochemically etching p-type silicon to an n-type etch stop. Data on etch rates at different $\mathrm{KOH}$ concentrations and temperatures and different silicon doping levels, as well as etch-stop mechanisms, have been documented by Seidel et al. [12], [13]. KOH and other orientation-dependent etches have been summarized by Williams [14].

While $\mathrm{KOH}$ is most often used for ODE of single-crystal silicon (SCS), it can also be used for rapid etching of shallow cavities of any shape, if the exact shape at the perimeter is not of concern. $\mathrm{KOH}$ has a high etch rate for both polycrystalline silicon and germanium, as well as for heavily ion-implanted SCS that has not been annealed.

$\mathrm{KOH}$ was found to etch silicon dioxides, LPCVD silicon nitride, and most of the transition metals tested slowly. High-refractive index PECVD nitride etched sufficiently slowly to be useful as a mask. Aluminum and photoresist were very rapidly etched. $\mathrm{KOH}$ is commonly masked with LPCVD silicon nitride, a sandwich of PECVD oxide and nitride (to reduce pinholes), or gold with a chromium adhesion layer.

10:1 HF: Mixed from 10 parts $\mathrm{H}_{2} \mathrm{O}: 1$ part $49 \% \mathrm{HF}$, by volume. $\mathrm{T} \approx 20{ }^{\circ} \mathrm{C}$ (room temperature). $\mathrm{HF}$ etches silicon dioxide. Such diluted HF solutions are used to strip thin layers of oxide and native oxide. The solution gets weaker with use. The etch rates of thermal oxide in various dilutions of HF are given in Table VIII. The etch rate is linear with concentration for dilutions in the 10:1 to 100:1 range.

5:1 BHF: Mixed from 5 parts $40 \% \mathrm{NH}_{4} \mathrm{~F}: 1$ part $49 \% \mathrm{HF}$, by volume. $\mathrm{T} \approx 20^{\circ} \mathrm{C}$ (room temperature). After mixing, buffered hydrofluoric acid (BHF) solutions should sit for several hours to stabilize, as the etch rate will initially be much higher than in equilibrium. Commercial mixes are also available. BHF, also known as buffered oxide etch (BOE), is used for its stable etch rate with use. The etch rates of thermal oxide in 5:1 and 10:1 BHF are also given in Table VIII. The etch rate in 10:1 BHF is exactly half that in 5:1 BHF.

Pad Etch 4: Pad Etch 4 from Ashland is a commercial mix of $11-15 \% \mathrm{NH}_{4} \mathrm{~F}+30-34 \% \mathrm{CH}_{3} \mathrm{COOH}+47-51 \% \mathrm{H}_{2} \mathrm{O}+4-8 \%$ propylene glycol + surfactant [15]. $\mathrm{T} \approx 20^{\circ} \mathrm{C}$ (room tempera- 
ture). This etchant is formulated to etch through a silicon dioxide dielectric layer, with the etch slowing greatly on aluminum pads or contacts. In these tests pure $\mathrm{Al}$ etched only slowly, but a long exposure was found to roughen the surface of $\mathrm{Al} / 2 \% \mathrm{Si}$, making it impossible to report an etch rate. Photoresist can be used as an etch mask for Pad Etch.

Phosphoric: Phosphoric Acid ( $85 \%$ by weight), $160{ }^{\circ} \mathrm{C}$. It is one of the few wet etches for silicon nitride. Because it etches photoresist, another masking material, such as oxide, must be used. The etch rates reported here for stoichiometric and silicon-rich LPCVD silicon nitrides are faster than reported earlier [3]. The etch rate is very sensitive to temperature, and this bath was likely a few degrees hotter. Hot phosphoric acid was also found to rapidly etch aluminum.

Al Etch A: Aluminum Etchant Type A from Transene is a commercial mix of $80 \% \mathrm{H}_{3} \mathrm{PO}_{4}+5 \% \mathrm{HNO}_{3}+5 \% \mathrm{CH}_{3} \mathrm{COOH}+$ $10 \% \mathrm{H}_{2} \mathrm{O}$ [16]. $\mathrm{T} \approx 50{ }^{\circ} \mathrm{C}$. It is formulated to selectively etch aluminum. It was also found to rapidly etch copper. Photoresist can be used as an etch mask for this etchant.

Ti Etch: Titanium wet etchant is mixed from 20 parts $\mathrm{H}_{2} \mathrm{O}: 1$ part $30 \% \mathrm{H}_{2} \mathrm{O}_{2}: 1$ part $49 \% \mathrm{HF}$, by volume. $\mathrm{T} \approx 20{ }^{\circ} \mathrm{C}$ (room temperature). Being a dilute HF solution, this etches oxides relatively slowly. Photoresist can be used as an etch mask for this etchant.

CR-7: Chromium etchant CR-7 from Cyantek is a commercial mix of $9 \%\left(\mathrm{NH}_{4}\right)_{2} \mathrm{Ce}\left(\mathrm{NO}_{3}\right)_{6}+6 \% \mathrm{HClO}_{4}+\mathrm{H}_{2} \mathrm{O}$ [17]. $\mathrm{T} \approx 20^{\circ} \mathrm{C}$ (room temperature). This etchant is formulated to selectively etch chromium, and was also found to etch copper and silver at useful rates. The manufacturer lists its shelf life as 12 months. Photoresist can be used as an etch mask for this etchant.

The chemical reaction for etching $\mathrm{Cr}$ is [18]

$$
\begin{aligned}
& 3 \mathrm{Ce}(\mathrm{IV})\left(\mathrm{NH}_{4}\right)_{2}\left(\mathrm{NO}_{3}\right)_{6(\mathrm{aq})}+\mathrm{Cr}_{(\mathrm{s})} \\
& \underset{\mathrm{H}^{+}}{\rightarrow} \mathrm{Cr}(\mathrm{III})\left(\mathrm{NO}_{3}\right)_{3(\mathrm{aq})}+3 \mathrm{Ce}(\mathrm{III})\left(\mathrm{NH}_{4}\right)_{2}\left(\mathrm{NO}_{3}\right)_{5(\mathrm{aq})}
\end{aligned}
$$

where the $\mathrm{H}^{+}$on the arrow indicates an acidic solution. The $\mathrm{Ce}^{4+}$ ions are reduced to $\mathrm{Ce}^{3+}$, while the $\mathrm{Cr}$ metal is oxidized to $\mathrm{Cr}^{3+}$. The etch products are soluble, and further reactions may occur in solution [18]. The perchloric acid is a stabilizer for the ceric ammonium nitrate.

CR-14: Chromium etchant CR-14 from Cyantek is a commercial mix of $22 \%\left(\mathrm{NH}_{4}\right)_{2} \mathrm{Ce}\left(\mathrm{NO}_{3}\right)_{6}+8 \% \mathrm{CH}_{3} \mathrm{COOH}+\mathrm{H}_{2} \mathrm{O}$ [19]. $\mathrm{T} \approx 20^{\circ} \mathrm{C}$ (room temperature). This etchant is also formulated to selectively etch chromium. It was also found to slowly etch vanadium and copper. When molybdenum was etched, it left behind a soft compound. The manufacturer lists its shelf life as 3 months. It etches chromium a little slower than CR-7, but over several years of use we found it to have more a consistent etch rate, as well as much less undercut of photoresist. The etch reaction is the same as for CR-7. The stabilizer is instead acetic acid.

Moly Etch: Molybdenum wet etchant is mixed from 180 parts $\mathrm{H}_{3} \mathrm{PO}_{4}: 11$ parts $\mathrm{CH}_{3} \mathrm{COOH}: 11$ parts $70 \% \mathrm{HNO}_{3}: 150$ parts $\mathrm{H}_{2} \mathrm{O}$, by volume. $\mathrm{T} \approx 20^{\circ} \mathrm{C}$ (room temperature). It can be masked with photoresist, which is etched at a moderate rate.

$\mathrm{H}_{2} \mathrm{O}_{2} 50{ }^{\circ} \mathrm{C}$ : Hydrogen peroxide ( $30 \%$ by weight). $\mathrm{T}=$ $50^{\circ} \mathrm{C}$. Hydrogen peroxide targets tungsten, but etches it slowly.
Heating increases the etch rate. Ultrasonic agitation (not done here) aids in etch uniformity by helping to remove bubbles. Warm $\mathrm{H}_{2} \mathrm{O}_{2}$ was also found to slowly etch chromium.

$\mathrm{Cu} \mathrm{FeCl} 3$ 200: Copper etchant type CE-200 from Transene is a commercial mix of $30 \% \mathrm{FeCl}_{3}+3-4 \% \mathrm{HCl}+\mathrm{H}_{2} \mathrm{O}$ [20]. $\mathrm{T} \approx 20^{\circ} \mathrm{C}$ (room temperature). When silver was placed in the solution, the film became thicker. CE-200 can be patterned with photoresist.

$\mathrm{FeCl}_{3}$ dissociates in water to generate $\mathrm{Fe}^{3+}$ and $\mathrm{Cl}^{-}$ions. One of the reactions for etching of copper with ferric chloride is [21]

$$
\mathrm{Cu}_{(\mathrm{s})}+2 \mathrm{Fe}^{3+}+3 \mathrm{Cl}^{-} \rightarrow \mathrm{CuCl}_{3}^{-}+2 \mathrm{Fe}^{2+} .
$$

Other chloride complexes are also formed. The $\mathrm{Fe}^{3+}$ ions are reduced to $\mathrm{Fe}^{2+}$, which remain in solution, while the copper metal is oxidized to $\mathrm{Cu}^{2+} . \mathrm{HCl}$ assists in the dissolution of the ferric chloride, and also etches copper itself [21]. The manufacturer recommends rinsing in a 5-10\% $\mathrm{HCl}$ solution after etching (not done here) before a final water rinse.

$\mathrm{Cu}$ APS 100: Copper etchant APS 100 from Transene is a commercial mix of 15-20\% $\left(\mathrm{NH}_{4}\right)_{2} \mathrm{~S}_{2} \mathrm{O}_{8}+\mathrm{H}_{2} \mathrm{O}$ [22]. $\mathrm{T}=$ $30^{\circ} \mathrm{C}$. Photoresist can be used as an etch mask for this etchant. The overall reaction for ammonium persulfate etching of copper is [21]

$$
\mathrm{Cu}_{(\mathrm{s})}+\left(\mathrm{NH}_{4}\right)_{2} \mathrm{~S}_{2} \mathrm{O}_{8(\mathrm{aq})} \rightarrow \mathrm{CuSO}_{4(\mathrm{aq})}+\left(\mathrm{NH}_{4}\right)_{2} \mathrm{SO}_{4(\mathrm{aq})} \cdot
$$

Dil. Aqua Regia: A diluted solution of aqua regia mixed from 3 parts $37 \% \mathrm{HCl}: 1$ part $70 \% \mathrm{HNO}_{3}: 2$ parts $\mathrm{H}_{2} \mathrm{O}$ (standard aqua regia does not have the additional water). The solution is self heating to about $30^{\circ} \mathrm{C}$ for tens of minutes for the shallow dish used in these tests. The water was added to the standard aqua regia solution to reduce the attack of photoresist. Aqua regia targets noble metals. In these tests, it was found to etch gold and palladium, but only slowly etch platinum (heating is require for a useful etch rate of $\mathrm{Pt}$ ). It was also found to etch nickel, copper, molybdenum, and aluminum. Because the $\mathrm{HCl}$ evaporates off when left open, it should be mixed just before use.

Gold reacts with chlorine, bromine, and iodine at elevated temperatures to form the corresponding halides, but reacts only slowly with fluorine [23]. At room temperature, the nitric acid in aqua regia is an oxidizing agent, while the addition of hydrochloric acid provides nascent chlorine to form a soluble etch product. Similar overall reactions are given in the literature [8] and [23], including [24]

$$
\begin{array}{r}
\mathrm{Au}_{(\mathrm{s})}+4 \mathrm{H}^{+}+\mathrm{NO}_{3}^{-}+4 \mathrm{Cl}^{-} \\
\end{array} \quad \rightarrow \mathrm{AuCl}_{4}^{-}+\mathrm{NO}_{(\mathrm{g})}+2 \mathrm{H}_{2} \mathrm{O}_{(\mathrm{l})} .
$$

The $\mathrm{HCl}$ in aqua regia can be replaced with $\mathrm{HBr}$ or $\mathrm{HI}$ [23], but not HF [8], as gold does not form a soluble fluoride [25]. Thus, HF-nitric solutions such as the silicon isotropic etch do not etch gold.

$A U$-5: Gold etchant AU-5 from Cyantek is a commercial mix of $5 \% \mathrm{I}_{2}+10 \% \mathrm{KI}+85 \% \mathrm{H}_{2} \mathrm{O}$ [26]. $\mathrm{T} \approx 20{ }^{\circ} \mathrm{C}$ (room temperature). It is formulated to selectively etch gold. Such iodine solutions are the color of dark coffee, making it impossible to 
observe a submerged sample. Rinsing with running water must be done to remove the solution from the surface. Isopropanol or methanol can be used for a faster rinse [18]. AU-5 can be patterned with photoresist. Rather than etching palladium, silver, and copper, it formed a compound that increased the film thickness above that of the original metal.

The overall reaction for etching gold is [18]

$$
2 \mathrm{Au}_{(\mathrm{s})}+\mathrm{I}_{2(\mathrm{aq})} \rightarrow 2 \mathrm{AuI}_{(\mathrm{aq})}
$$

The potassium iodide increases the solubility of both the iodine and gold(I) iodide in water [18], [27], allowing a greater concentration of reactant in the solution and the etch product to be removed so that the etch can proceed.

$\mathrm{NiCr}$ TFN: Nichrome etchant TFN from Transene is a commercial mix of $\left.10-20 \%\left(\mathrm{NH}_{4}\right)_{2} \mathrm{Ce}\left(\mathrm{NO}_{3}\right)_{6}\right)+5-6 \%$ $\mathrm{HNO}_{3}+\mathrm{H}_{2} \mathrm{O}$ ) [28]. $\mathrm{T} \approx 20{ }^{\circ} \mathrm{C}$ (room temperature). It is formulated to etch nickel-chromium alloy. It etched pure nickel slowly, but etched chromium, copper, and molybdenum faster than the NiCr alloy. Photoresist can be used as an etch mask for this etch.

Phos + Sulf: A mix of 1 part $96 \% \mathrm{H}_{2} \mathrm{SO}_{4}: 1$ part $85 \% \mathrm{H}_{3}$ $\mathrm{PO}_{4} \cdot \mathrm{T}=160{ }^{\circ} \mathrm{C}$. This solution is listed as an etchant for sapphire [1], [2], but did not etch it even at this high temperature.

Piranha: The Piranha (also known as sulfuric-peroxide) used here is a mix of $\sim 50$ parts $96 \% \mathrm{H}_{2} \mathrm{SO}_{4}: 1$ part $30 \% \mathrm{H}_{2} \mathrm{O}_{2}$. $\mathrm{T}=120{ }^{\circ} \mathrm{C}$. The hydrogen peroxide is added just before use. It is used as a cleaning solution that strips organics and some metals. In these tests, it was indeed found to etch photoresist, the resist pen, and polyimide very rapidly, but only slowly attacked the Parylene C. It also etched aluminum, nickel, and silver rapidly. Chromium was etched slowly, which allows it to be used for the cleaning of photomasks.

Microstrip: Arch Microstrip 2001 is a commercial photoresist stripper mixed from 45-55\% n-methyl pyrrolidone and $40-55 \%$ 2-(2-aminoethoxy)ethanol [29]. $\mathrm{T}=85^{\circ} \mathrm{C}$. It is formulated to strip resists without affecting structural materials. In these tests, it very rapidly removed the photoresists and the resist pen, while etching the polyimide at a moderate rate and not etching graphite.

Acetone: Pure acetone, also known as dimethyl ketone. It is used to strip photoresist and for cleaning off organics. It removed photoresists rapidly, as expected, but did not etch polyimide.

Methanol: Pure methanol. It is used for cleaning and in supercritical drying. Like acetone, methanol removed photoresist, but did not etch polyimide.

Isopropanol: Pure isopropanol, also known as isopropyl alcohol (IPA) and 2-propanol. It is used for cleaning off organics. In contrast to methanol, isopropanol was found to remove photoresist at a moderate rate.

\section{B. Dry Etches}

Most dry etches employ a plasma to generate reactive species, but several plasmaless-vapor-phase etches have been used in the fabrication of MEMS.
$\mathrm{XeF}_{2}$, 2.6 mtorr, Homemade Chamber: Xenon difluoride gas (no plasma) from a solid source at its full vapor pressure at room temperature. It targets silicon, as well as metals that form volatile fluorides such as $\mathrm{Ti}, \mathrm{W}, \mathrm{Mo}$, and Ta [30]. It does not etch silicon dioxide, aluminum, or photoresist, and only slowly etches silicon nitride. Commercial $\mathrm{XeF}_{2}$ etchers are now available. Other noble-gas fluorides have also been used to etch silicon [14].

$H F$ Vapor Over Dish: $\mathrm{HF}+\mathrm{H}_{2} \mathrm{O}$ vapor evaporated at room temperature from liquid $49 \% \mathrm{HF} / \mathrm{H}_{2} \mathrm{O}$, with wafer continuously exposed $\sim 1 \mathrm{~cm}$ over the dish. Like liquid HF, HF vapor targets silicon dioxides. Continuous exposure to the vapor results in droplet condensation on the wafer after about $25 \mathrm{~s}$ at room temperature, with the underlying areas having higher oxide etch rate. "Pulsed" etching, in which the wafer is alternately held over the liquid for $15 \mathrm{~s}$, then removed and optionally heated for $15 \mathrm{~s}$, eliminates droplet formation. Simply heating the wafer while over the $\mathrm{HF} / \mathrm{H}_{2} \mathrm{O}$ also inhibits condensation, while still allowing etching to occur. In these tests, photoresist survived etching, but peeled when rinsed.

Manual vapor etching of oxide is particularly useful in cases where fragile structures cannot tolerate loading into a plasma etcher and where high selectivity is needed. Other users of this etch have found a nonvolatile residue left behind when doped oxides such as PSG [31] or silicon oxynitride [32] are exposed to HF vapor. Titanium and copper have been found to form nonvolatile fluoride layers [31]. While we found aluminum to be attacked, it has also been found to form a thick fluoride film when anhydrous $\mathrm{HF}+\mathrm{H}_{2} \mathrm{O}$ was used under more controlled conditions [31]. Commercial HF-vapor etchers are now available.

Technics $\mathrm{O}_{2}$ : Technics West PE II-A plasma etcher using the recipe $\mathrm{O}_{2}$ flow rate $\approx 51 \mathrm{sccm}$, power $(\mathrm{P})=400 \mathrm{~W}$ at $30 \mathrm{kHz}$, pressure $(p) \approx 300$ mtorr. Oxygen plasmas are used for stripping and descumming photoresist, as well as cleaning off other organics. In these tests, the photoresists, Parylene, and polyimide etched at about the same rate, but the graphite was not etched.

DRIE HF Mech: Surface Technology Systems (STS) Advanced Silicon Etch (ASE) inductively coupled plasma DRIE tool, using a typical Bosch-process recipe for nearly vertical trenches with high platen frequency [33], [34]: etch for $6 \mathrm{~s}$ with $\mathrm{SF}_{6}=80 \mathrm{sccm}$, coil $\mathrm{P}=600 \mathrm{~W}$ at $13.56 \mathrm{MHz}$, platen $\mathrm{P}=12$ $\mathrm{W}$ at $13.56 \mathrm{MHz}, \mathrm{p} \approx 12$ mtorr; then passivate for $5 \mathrm{~s}$ with $\mathrm{C}_{4} \mathrm{~F}_{8}=80 \mathrm{sccm}$, coil $\mathrm{P}=600 \mathrm{~W}$ at $13.56 \mathrm{MHz}$, platen $\mathrm{P}=0$ $\mathrm{W}, \mathrm{p} \approx 8$ mtorr. The etching and passivation steps are alternated. The platen structure has $20^{\circ} \mathrm{C}$ coolant flowing through it, while the wafer heats to about $65^{\circ} \mathrm{C}$ during an etch. The wafer clamping in this particular etcher, required due to backside helium pressure for improved heat transfer, is mechanical.

This etcher and recipe are designed for deep, near-vertical etching of silicon. The etch rate of silicon is a strong function of the area of silicon exposed and the trench aspect ratio (depth/width) for deep etches, slowing with increases in each. The etch rate also tends to be noticeably slower at the center of the wafer. In these tests, a higher-than-typical area of $~ 50 \%$ was exposed. Due to a relatively short etch time, only shallow trenches were formed. Experience with several such etchers and chucks (with either mechanical or electrostatic wafer clamping) 
shows that the etch rate and profile are highly variable with equipment. Recipes are usually not transferable from one machine to another and can be affected by seemingly small changes in the setup of an etcher.

Photoresist and oxide have been used as masks for DRIE. These tests confirmed very high selectivity of etching silicon over photoresist and silicon dioxide, and also found high selectivity over silicon nitride. The manufacturer recommends not using metal masks. Several metals with volatile fluorides were tested and found to etch slowly with this recipe.

DRIE HF ES: Surface Technology Systems ASE inductively coupled plasma DRIE tool, using a typical Bosch-process recipe for nearly vertical trenches with high platen frequency: etch for $9 \mathrm{~s}$ with $\mathrm{SF}_{6}=130 \mathrm{sccm}, \mathrm{O}_{2}=13 \mathrm{sccm}$, coil $\mathrm{P}=600 \mathrm{~W}$ at 13.56 MHz, platen $\mathrm{P}=12 \mathrm{~W}$ at $13.56 \mathrm{MHz}$; then passivate for $7 \mathrm{~s}$ with $\mathrm{C}_{4} \mathrm{~F}_{8}=85 \mathrm{sccm}$, coil $\mathrm{P}=600 \mathrm{~W}$ at $13.56 \mathrm{MHz}$, platen $\mathrm{P}=0 \mathrm{~W} ; \mathrm{APC}=66 \%$; with electrostatic wafer clamping.

DRIE LF Mech: Surface Technology Systems ASE inductively coupled plasma DRIE tool, using a typical low-platen frequency, stop-on-oxide recipe for nearly vertical trenches [35]: etch for $5 \mathrm{~s}$ with $\mathrm{SF}_{6}=125 \mathrm{sccm}$, coil $\mathrm{P}=600 \mathrm{~W}$ at 13.56 MHz, platen $\mathrm{P}=30 \mathrm{~W}$ at $380 \mathrm{kHz}$ pulsed on and off on the order of 10 milliseconds (the exact timing is proprietary), $\mathrm{p} \approx 28$ mtorr; then passivate for $6.7 \mathrm{~s}$ with $\mathrm{C}_{4} \mathrm{~F}_{8}=150 \mathrm{sccm}$, coil $\mathrm{P}=600 \mathrm{~W}$ at $13.56 \mathrm{MHz}$, platen $\mathrm{P}=0 \mathrm{~W}, \mathrm{p} \approx 28 \mathrm{mtorr}$; with mechanical wafer clamping.

DRIE LF ES: Surface Technology Systems ASE inductively coupled plasma DRIE tool, using a typical low-platen frequency, stop-on-oxide recipe to greatly reduce notching, for nearly vertical trenches: etch for $8 \mathrm{~s}$ with $\mathrm{SF}_{6}=80 \mathrm{sccm}$, coil $\mathrm{P}=600 \mathrm{~W}$ at $13.56 \mathrm{MHz}$, platen $\mathrm{P}=30 \mathrm{~W}$ at $380 \mathrm{kHz}$ pulsed on and off on the order of milliseconds (the exact timing is proprietary), $\mathrm{p} \approx 12 \mathrm{mtorr}$; then passivate for $5 \mathrm{~s}$ with $\mathrm{C}_{4} \mathrm{~F}_{8}=80 \mathrm{sccm}$, coil $\mathrm{P}=600 \mathrm{~W}$ at $13.56 \mathrm{MHz}$, platen $\mathrm{P}=0 \mathrm{~W}, \mathrm{p} \approx 8$ mtorr; with electrostatic wafer clamping.

STS 320 SF $_{6}$ : Surface Technology Systems 320 parallelplate RIE tool, using the recipe $\mathrm{SF}_{6}=25 \mathrm{sccm}, \mathrm{P}=100 \mathrm{~W}$ at $13.56 \mathrm{MHz}, p=20$ mtorr. The driven electrode area is about $900 \mathrm{~cm}^{2}$. This etch targets silicon, silicon nitride, and transition metals with volatile fluorides. Photoresist can be used as an etch mask for this etch.

STS $\mathrm{SF}_{6}+\mathrm{O}_{2}$ : Surface Technology Systems 320 parallel-plate RIE tool, using the recipe $\mathrm{SF}_{6}=25 \mathrm{sccm}$, $\mathrm{O}_{2}=10 \mathrm{sccm}, \mathrm{P}=100 \mathrm{~W}$ at $13.56 \mathrm{MHz}, \mathrm{p}=20$ mtorr. The driven electrode area is about $900 \mathrm{~cm}^{2}$. This etch targets silicon, silicon nitride, and transition metals with volatile fluorides. The addition of oxygen increases the etch rate of the target materials, but also increases the etch rate of the photoresist, which can reduce the selectivity.

STS $320 \mathrm{CF}_{4}$ : Surface Technology Systems 320 parallelplate RIE tool, using the recipe $\mathrm{CF}_{4}=25 \mathrm{sccm}, \mathrm{P}=100 \mathrm{~W}$ at $13.56 \mathrm{MHz}, \mathrm{p}=60 \mathrm{mtorr}$. The driven electrode area is about $900 \mathrm{~cm}^{2}$. This etch targets silicon dioxide, which can be patterned with photoresist or a nonvolatile metal such as chromium. This recipe results in fluorocarbon polymer buildup on the chamber walls and a reduced etch rate over time. A small amount of fluorocarbon residue left on the wafer can affect the later formation of electrical contacts if it is not removed.
TABLE IX

Etch Rates of Gold Deposited by Three Methodsin Two ETCHANTS $(\mathrm{nm} / \mathrm{min})$

\begin{tabular}{l|r|r|r}
\hline Etchant & $\begin{array}{c}\text { Ion-Milled } \\
\text { Gold } \\
\text { (at 1000 V) }\end{array}$ & $\begin{array}{c}\text { Evaporated } \\
\text { Gold } \\
\text { (at } 0.5 \mathrm{~nm} / \mathrm{s})\end{array}$ & $\begin{array}{r}\text { Electroplated } \\
\text { Gold }\left(\mathrm{NaAuSO}_{3},\right. \\
40 \mathrm{~mA} / \mathrm{cm} 2)\end{array}$ \\
\hline Cyantek AU-5 & 490 & 660 & 560 \\
\hline Dilute aqua regia & 530 & 680 & 1000 \\
\hline
\end{tabular}

STS $\mathrm{CF}_{4}+\mathrm{O}_{2}$ : Surface Technology Systems 320 parallelplate RIE tool, using the recipe $\mathrm{CF}_{4}=25 \mathrm{sccm}, \mathrm{O}_{2}=5 \mathrm{sccm}$, $\mathrm{P}=100 \mathrm{~W}$ at $13.56 \mathrm{MHz}, \mathrm{p}=60$ mtorr. The driven electrode area is about $900 \mathrm{~cm}^{2} . \mathrm{CF}_{4}$-based etches typically target silicon dioxide, but this etch was found to etch silicon nitride and silicon even faster than oxide. The addition of oxygen in this recipe increases the oxide etch rate but also increases the photoresist etch rate, significantly reducing the selectivitiy. The oxygen also reduces fluorocarbon polymer buildup on the chamber walls, yielding a more stable etch rate over time.

Ion Mill: Commonwealth Scientific ion mill, ion milling with argon ions at $500 \mathrm{~V}$, current density of $\sim 1 \mathrm{~mA} / \mathrm{cm}^{2}$, chamber $\mathrm{p}=2 \times 10^{-4}$ torr, at an angle normal to the wafer. Ion milling with an inert gas such as argon is a purely physical process, so any material can be etched by this method. The surface of unpatterned S1822 photoresist became wavy after a 10-min etch but retained most of its thickness, so this photoresist may not be a useable masking material.

\section{Etch-Rate Variation}

Many reasons for etch-rate variation were enumerated earlier [3], [14]. One of the most significant is the properties of the material, which are a result of the production method and subsequent processing (e.g., annealing). This is apparent by inspection of Table III: materials that are primarily silicon dioxide have widely varying etch rates in 5:1 BHF and other etchants.

As another demonstration of the effect of deposition conditions, gold was deposited using three different methods: ion milling and evaporation, as described in Section II, and by electroplating from a sodium gold sulfite bath at current density of $40 \mathrm{~mA} / \mathrm{cm}^{2}$. The etch rates in the AU-5 and dilute aqua regia solutions are given in Table IX. Ion-milled gold, which is the densest, has the lowest etch rate in both etchants. Electroplated gold, known for having a lower density than the bulk metal, has a significantly higher etch rate in dilute aqua regia.

Another example of etch-rate variation with material deposition method is given in Table X. PECVD silicon nitride was deposited using the recipe described in Section II, but with varying flow rates of $2 \% \mathrm{SiH}_{4} / \mathrm{Ar}$. For the lowest silane flow rates, the silicon content is probably lowest, which agrees with the low index of refraction. These films have the highest etch rates in 10:1 BHF. Higher silane flow rates yield increasingly higher indexes of refraction and lower etch rates. The lowest etch rate, $2.5 \mathrm{~nm} / \mathrm{min}$, is still much faster than for LPCVD silicon nitride (which has much less hydrogen). 
TABLE $X$

PECVD SILICON NITRIDE INDEXES OF REFRACTION AND ETCH RATES IN 10:1 BHF (nm/min)

\begin{tabular}{r|r|r}
\hline $\begin{array}{c}2 \% \text { Si in Ar } \\
(\mathrm{sccm})\end{array}$ & $\begin{array}{c}\text { Index of } \\
\text { Refraction }\end{array}$ & $\begin{array}{c}\text { Etch Rate } \\
\text { in 10:1 BHF } \\
(\mathrm{nm} / \mathrm{min})\end{array}$ \\
\hline 70 & 1.83 & 11 \\
\hline 100 & 1.88 & 7.3 \\
\hline 200 & 2.03 & 4.3 \\
\hline 500 & 2.53 & 2.7 \\
\hline 750 & 2.57 & 2.8 \\
\hline 1000 & 2.67 & 2.5 \\
\hline
\end{tabular}

\section{Etch-Rate Measurement Techniques}

All films were coated onto 100-mm silicon wafers. The transparent films, opaque films, and wafer substrates required different measurement techniques.

Most of the data presented here has not been reported before. In the cases in the tables for which new data was not taken, etch rates were transferred from our earlier paper [3]. In case of discrepancies, the newer data is reported. Most of the ion-mill data for the elements is from Commonwealth Scientific [36] and agrees with our few measurements. This outside data is included due to the usefulness of this tool.

Measured etch rates are rounded to two significant figures.

\section{A. Transparent Films}

The transparent films in these tests are polycrystalline silicon, silicon dioxide, silicon nitride, alumina, photoresist, parylene, and polyimide. Refractive indexes (RIs) were measured with a Gaertner Scientific ellipsometer at $632.8 \mathrm{~nm}$ for most films (the RI of polysilicon is already known). These RIs were used in interferometric thickness measurements using a NanoSpec AFT tool. Polycrystalline silicon was deposited on a wafer that already had $100 \mathrm{~nm}$ of thermal oxide to give reflections that could be detected by the NanoSpec.

To reduce the number of samples to be prepared, some wafers were divided into quarters. For wet etches and dry etches with manual wafer loading, the quarter wafers were used. At least three thickness measurements were made before and after etching. Etching was done without masking. For dry etches with automatic wafer loading, a whole wafer was used and five points were measured. The average thickness difference, divided by the time, gives the etch rate.

\section{B. Opaque Films and Wafers}

The opaque films used in these tests are graphite, the metals, titanium nitride, and the resist pen. Ge and SiGe films, while not opaque, were measured with this group. The wafers are single-crystal silicon, fused quartz, Pyrex 7740, and sapphire. These samples could not be measured by the simpler interferometric method, and a calibrated Dektak II profilometer was used. In most cases, the wafer had photoresist applied and patterned, with about $50 \%$ of the wafer area exposed. In a few cases, the resist pen was used to make a mask. For testing the photoresists and resist pen themselves, the photoresist was patterned and ink was simply drawn onto a wafer known not to etch. For cases in which the etchant is known to rapidly etch photoresist (e.g., in $\mathrm{KOH}$ and Piranha), a mask of evaporated $\mathrm{Cr} / \mathrm{Au}$ was used. For cases in which a silicon wafer is known to etch rapidly (e.g., in silicon isotropic etchant and $\mathrm{KOH}$ ), the wafers were first coated with LPCVD silicon nitride to protect both sides.

Etches were performed, the photoresist (or $\mathrm{Cr} / \mathrm{Au}$ ) was stripped off, and at least three step heights were measured. The average step height divided by the time gives the etch rate.

For very rapid etches, step heights were measured where the removal of Kapton tape after deposition had left a blank spot on the wafer. The materials were etched until visibly gone and the time recorded. The initial thickness divided by the time gives the etch rate.

\section{Etch Times}

Etching times were selected to give a thickness change typically in the range of 0.1 to $1 \mu \mathrm{m}$ to give good confidence to the results.

As expected, however, many samples etched very slowly. In these cases, the etches were performed for at least $20 \mathrm{~min}$ to give a reasonable step height or interferometric thickness change, if any. For the samples measured by profilometer, when it was impossible to see a clear step amidst the roughness, an upper limit of etch rate (e.g., $<2 \mathrm{~nm} / \mathrm{min}$ ) is given. This is calculated by dividing the roughness by the etch time.

In some other cases, the etch was so rapid (e.g., photoresist in Piranha and acetone) that it was impossible to tell exactly when the film was gone due to clouds or bubbles blocking view of the film. For these cases, a lower limit of the etch rate is reported (e.g., $>92000 \mathrm{~nm} / \mathrm{min}$ ). This is calculated by dividing the initial thickness by the time at which the clouds or bubbles had dissipated enough to view the cleared wafer.

\section{ETCH-RATE RESUltS AND Discussion}

Etch rates were reported in Tables II-VII. Selectivities can readily be calculated from these data.

Not all materials were etched in all etchants due to time limitations and contamination concerns with the plasma etchers. Certain materials commonly used in micromachining were considered key materials (undoped polysilicon, thermal silicon dioxide, stoichiometric LPCVD silicon nitride, $\mathrm{Al} / 2 \% \mathrm{Si}$, chromium, and S1822 photoresist) and were etched in most etchants in order to determine their compatibility. Similarly, certain etches (isotropic silicon etchant, potassium hydroxide, 5:1 BHF, CR-7 chromium etchant, Piranha, ion milling, and $\mathrm{SF}_{6}+\mathrm{O}_{2}$, and $\mathrm{CF}_{4}+\mathrm{O}_{2}$ plasmas) were considered to be key etchants and most materials were etched in them.

One of the realities of putting materials in etchants intended to etch a different material is that a clear result is not attained. In many cases materials were roughened, but little etching apparently occurred. The degree of roughening probably depends on the microstructure (i.e., grain size and dislocation density) and thus varies with the method of material preparation.

At the bottom of Table I is a summary of the letter notations used in the tables. In cases where etch rates were not measured by the authors, but the etches are known from experience with 
similar materials or from the literature to work, be relatively fast (faster than $\sim 500 \mathrm{~nm} / \mathrm{min}$ ), or be slow (slower than $\sim 10$ $\mathrm{nm} / \mathrm{min}$ ) or have zero etch rate, the notations $W, F$, or $S$, respectively, are used. If the film was roughened or attacked, the notation is $R$. This may include an upper limit of etch rate (e.g., $<1 \mathrm{~nm} / \mathrm{min}$ ) if an exact value could not be determined. If the film was thicker after being exposed to the etch, the notation is $T$. This occurred with the photoresists absorbing some of the liquid and swelling, and in other cases in which an insoluble compound formed. If the film peeled during the etch or rinsing, the notation is $P$. Some etches initially had a very slow etch rate during an "incubation time," and are so noted by $I$. Films that congealed are noted with $C$. Films that remained after exposure to the etch, but were soft have a notation of soft.

\section{A. Discussion of the Etches}

Intended uses of the etches and specific information about the materials were given in Sections II and III. More general observations are given here.

$\mathrm{KOH}$ did not appreciably etch most of the transition metals and the silicon nitrides, and had a zero etch rate for Mo, W, $\mathrm{Ni}, \mathrm{Pd}, \mathrm{Pt}$, and $\mathrm{Au}$. These might be used in micromachining as structural materials, with the underlying silicon substrate being removed in $\mathrm{KOH}$.

The HF solutions, 5:1 BHF and 10:1 HF, did not appreciably etch most of the transition metals, and had a zero etch rate for $\mathrm{Nb}, \mathrm{Cr}, \mathrm{Ta}, \mathrm{Pd}, \mathrm{Pt}$, and Au. The exception is Ti, which etches even faster than the silicon dioxides in 10:1 HF. Thus, $\mathrm{Cr}$, $10 \mathrm{Ti} / 90 \mathrm{~W}$, or TiN should be used rather than Ti as an adhesion layer in the presence of HF. The metals with a slow HF etch rate might be used in surface micromachining as structural materials, with silicon dioxide being the sacrificial layer.

Piranha removed most metals at moderate to fast rates. $\mathrm{Nb}$, $\mathrm{Ta}, \mathrm{Cr}, \mathrm{Mo}, \mathrm{Pd}, \mathrm{Au}$, and $10 \mathrm{Ti} / 90 \mathrm{~W}$ were etched only slowly (Au not at all), allowing the use of Piranha for cleaning with these metals present.

Microstrip 2001 and acetone dissolved the resists tested rapidly. Methanol removed some resists and made a mess of others, causing them to peel or congeal. Isopropanol had much less effect, with the slow removal of resist.

The oxygen plasma only etched the organics, but did not measurably etch graphite.

The STS DRIE's etched silicon and germanium much faster than the oxides, nitrides, and photoresists, making the latter useful as masking layers. The transition metals tested, all of which have volatile fluorides, etched only slowly.

The $\mathrm{SF}_{6}+\mathrm{O}_{2}$ plasma etch etched silicon the fastest, the silicon nitrides at a moderate rate, and the silicon dioxides slowly. Moderate etch rates were also measured for Mo and $10 \mathrm{Ti} / 90$ W. Other metals, which do not have volatile etch products, were removed slowly due to sputtering.

The $\mathrm{CF}_{4}+\mathrm{O}_{2}$ plasma etched the silicon nitrides faster than silicon or oxide, but at only a moderate rate. For the metals tested, molybdenum had a moderate etch rate, and other metals again were removed slowly due to sputtering.

Ion milling can remove any material due to its purely physical nature, and is thus thought often of as having roughly the same etch rate for different materials. The data, however, show that there actually is a wide range of etch rates such that some materials can be used as etch masks for others. Graphite is etched very slowly. The metals $\mathrm{Ag}, \mathrm{Au}, \mathrm{Pd}, \mathrm{Cu}$, and $\mathrm{Pt}$ are all etched more than 20 times faster.

\section{B. Discussion of the Materials}

Changes in the deposition method of pure materials, as well as the addition of small amounts of impurities, tend to produce much greater differences in etch rate for wet etchants than for plasma etching. Comparing the etch rates of the various silicon nitrides in the 5:1 BHF and $\mathrm{SF}_{6}+\mathrm{O}_{2}$ plasma illustrates this point, with the ratio of fastest- to slowest-etching nitride in BHF being 46 , but only 1.3 for the plasma etch. Comparing the etch rates of the many silicon dioxide samples in 5:1 BHF and in $\mathrm{CF} 1_{4}+\mathrm{O}_{2}$ plasma gives similar results.

PECVD oxides and nitride etch faster in most etchants than their LPCVD counterparts. Annealing both PECVD and LPCVD oxides reduces the etch rate in HF solutions. Annealed LTO has similar etching properties to thermal oxide.

Unlike sapphire, the aluminum oxide films were etched in most of the wet etchants tested.

Samples of patterned $\mathrm{Cr}$ on top of $\mathrm{Au}$ were produced to study a possible electrochemical effect. This $\mathrm{Cr}$ etched slower in CR-7, but faster in CR-14, as compared to evaporated $\mathrm{Cr}$ on $\mathrm{Si}$. In this particular test, $\mathrm{Cr}$ on Au peeled off in 5:1 BHF, but we have successfully used $\mathrm{Cr}$ as an adhesion layer for $\mathrm{Au}$ in surface-micromachined devices in which the $\mathrm{Cr}$ was exposed.

Silver grew thicker in AU-5 and in $\mathrm{Cu} \mathrm{FeCl}_{3} 200$ etchant, perhaps due to the formation of insoluble compounds such as AgI.

Metals deposited by evaporation, in which the atoms forming the film arrive with low energy, tend to etch faster than the denser films deposited by sputtering and by ion milling, with high-incident-energy atoms.

TiN became soft in silicon isotropic etchant. Titanium became soft in KOH. Molybdenum became soft in CR-14. The etch rates reported are based on the difference between the initial thickness and the thickness of the remaining material.

Polyimide stands up to wet etchants better than the resists, but has similar etch rates in the plasma etches. Parylene-C did not etch appreciably in the wet etchants.

\section{CONCLUSION}

The data in the tables show useful etchants for most materials, as well as indicating in which etchants the materials will survive. They can also be used to calculate selectivities to choose masking layers should photoresist not be sufficient.

For surface micromachining, the traditional system of materials and etchant is a structural material of polysilicon, an underlying layer of polysilicon, a sacrificial layer based on silicon dioxide, silicon nitride, or silicon wafer, and an HF-based etchant. These data show that a number of other materials might be substituted as the structural layer: LPCVD silicon nitride, V, $\mathrm{Nb}, \mathrm{Ta}, \mathrm{Cr}, \mathrm{Mo}, \mathrm{W}, \mathrm{Pd}, \mathrm{Pt}, \mathrm{Cu}, \mathrm{Ag}, \mathrm{Au}, 90 \mathrm{Ti} / 10 \mathrm{~W}, 80 \mathrm{Ni} / 20 \mathrm{Cr}$, or polyimide. For bulk micromachining of a silicon wafer with $\mathrm{KOH}$ to leave a freestanding film, many of the same materials 
might be used: silicon nitride, $\mathrm{Nb}, \mathrm{Ta}, \mathrm{Cr}, \mathrm{Mo}, \mathrm{W}, \mathrm{Ni}, \mathrm{Pd}, \mathrm{Pt}$, and $\mathrm{Au}$. Other systems might be used, such as a sacrificial layer of $\mathrm{Al}$ etched with Aluminum Etchant Type A, and a structural layer of PECVD silicon dioxide, Ti, or Cr. Another possibility is a sacrificial layer of polysilicon or single-crystal silicon etched with $\mathrm{XeF}_{2}$, and a structural layer of $\mathrm{SiO}_{2}, \mathrm{Au}$, or Pt. Many more systems can be deduced from the data.

Alternative materials systems for MEMS will be of interest in the future for many reasons: A previously unused structural material will be desired, for example, for its chemical inertness, for its high or low Young's modulus, for its high or low thermal conductivity, for its low deposition temperature, or for its biological compatibility. These data will aid in selecting appropriate etchants, substrates, and coexisting materials.

\section{ACKNOWLEDGMENT}

The authors would like to thank A. W. Flounders, Technology Manager, and N. Chen, Assistant Development Engineer, of the U.C. Berkeley Microfabrication Laboratory, and the Berkeley Sensor \& Actuator Center for preparation of some of the samples. They also thank Agilent Laboratories management for summer-student funding for K. Gupta, and Q. Bai of Agilent Labs for assistance with a DRIE test.

\section{REFERENCES}

[1] J. L. Vossen and W. Kern, Eds., Thin Film Processes. New York, NY: Academic, 1978, ch. V-1.

[2] CRC Handbook of Metal Etchants, CRC, Boca Raton, FL

[3] K. R. Williams and R. S. Muller, "Etch rates for micromachining processing," J. Microelectromech. Syst., vol. 5, pp. 256-269, Dec. 1996.

[4] A. E. Franke et al., "Post-CMOS modular integration of poly-SiGe microstructures using poly-Ge sacrificial layers," in Tech. Dig. SolidState Sensor and Actuator Workshop, Hilton Head, SC, June 2000 pp. 18-21.

[5] A. C. Adams, "Silicon nitride and other insulator films," in Plasma Deposited Thin Films, J. Mort and F. Jansen, Eds. Boca Raton, Florida: CRC, 1986, ch. 5 .

[6] R. C. Ruby et al., "Thin film bulk wave acoustic resonators (FBAR) for wireless applications," Proc. 2001 IEEE Ultrasonics Symposium, vol. 1 pp. 813-821, Oct. 2001

[7] K. R. Williams and R. S. Muller, "Micromachined hot-filament ionization pressure sensor and magnetometer," in Tech. Dig. 1997 Int. Conf Solid-State Sensors and Actuators (Transducers'97), Chicago, IL, June 1997, pp. 1249-1252.

[8] C. A. Hampel, The Encyclopedia of the Chemical Elements. New York: Reinhold Book Corp., 1968.

[9] T. Pornsin-Sisirak et al., "MEMS wing technology for a battery-power ornithopter," Proc. Thirteenth IEEE Int. Conf. on Micro Electro Mechanical Systems (MEMS'00), pp. 799-804, Jan. 2000.

[10] B. Schwartz and H. Robbins, "Chemical etching of silicon, IV. Etching technology," J. Electrochem. Soc., vol. 123, no. 12, pp. 1903-1910, Dec. 1976.

[11] G. Wulfsberg, Inorganic Chemistry. Sausalito, CA: University Science Books, 2000

[12] H. Seidel et al., "Anisotropic etching of crystalline silicon in alkaline solutions, I. Orientation dependence and behavior of passivation layers," J. Electrochem. Soc., vol. 137, no. 11, pp. 3612-3626, Nov. 1990.

[13] H. Seidel et al., "Anisotropic etching of crystalline silicon in alkaline solutions, II. Influence of dopants," J. Electrochem. Soc., vol. 137, no. 11, pp. 3626-36232, Nov. 1990.

[14] K. R. Williams, "Etching of Single-Crystal Silicon," in Properties of crystalline silicon, R. Hull, Ed. London, U. K.: INSPEC/IEE, 1999, ch. 16.
[15] Material Safety Data Sheet for Pad Etch 4, 1998. Ashland Specialty Chemical Co.

[16] Material Safety Data Sheet for Aluminum Etchant Type A, 1987. Transene Co. Inc.

[17] Material Safety Data Sheet for CR-7, 2001. Cyantek Corp..

[18] A. Salehi, private communication, Mar. 2003.

[19] Material Safety Data Sheet for CR-14, 1998. Cyantek Corp.

[20] Material Safety Data Sheet for Copper Etchant Type 100/200, 1986. Transene Co., Inc.

[21] C. Christuk, private communication, Apr. 2003.

[22] Material Safety Data Sheet for Copper Etch APS-100, 2002. Transene Co., Inc..

[23] Handbook of Inorganic Chemistry, Mc-Graw Hill, New York, NY, 2003.

[24] D. F. Shriver and P. W. Atkins, Inorganic Chemistry. Oxford, U.K. Oxford University Press, 1999.

[25] N. V. Sidgwick, The Chemical Elements and Their Compounds. Oxford, U.K.: Clarendon Press, 1950

[26] Material Safety Data Sheet for AU-5, 2001. Cyantek Corp.

[27] R. C. Weast, Ed., CRC Handbook of Chemistry and Physics, 66 ed. Boca Raton, FL: CRC, 1985, pp. B98-B101.

[28] Material Safety Data Sheet for Chromium Etchant Type 1020, Chrome Etch $8001 N$, and Nichrome Etch TFN, 2002. Transene Co., Inc..

[29] Material Safety Data Sheet for Microstrip 2001, 1999. Arch Chemicals, Inc..

[30] Description of $\mathrm{XeF}_{2}$ MEMS Etcher [Online]. Available: www.pentavacuum.com/mems.htm

[31] R. Hanestad et al., "Stiction-free release etch with anhydroud HF/water vapor processes," in Proc. SPIE: Micromachining and Microfabrication Process Technology VII, vol. 4557, San Francisco, CA, Oct. 2001, pp. $58-68$.

[32] S. Hoen, private communication, 2000 personal communication.

[33] F. Laermer et al., "Method for Anisotropic Plasma Etching of Substrates,", Mar. 12, 1996.

[34] A. A. Ayon et al., "Etching characteristics and profile control in a time multiplexed inductively coupled plasma etcher," in Tech. Dig. SolidState Sensor and Actuator Workshop, Hilton Head, SC, June 1998, pp. $41-44$.

[35] J. Hopkins et al., "Method and Apparatus for Etching a Substrate,", Feb. $13,2001$.

[36] H. R. Kaufman and R. S. Robinson, Operation of Broad-Beam Sources. Alexandria, VA: Commonwealth Scientific Corporation, 1984, p. 109.

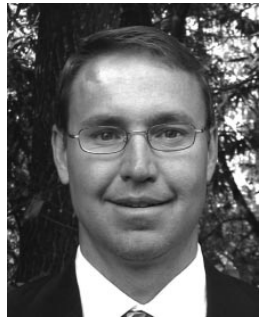

Kirt R. Williams (S'90-M'97-SM'01) received the B.S. degree with high honors with a double major in electrical engineering and computer sciences (EECS) and materials science and engineering from the University of California, Berkeley, in 1987. He received the M.S. and Ph.D. degrees in electrical engineering and computer science from the University of California at Berkeley in 1993 and 1997, respectively.

After receiving the B.S. degree, he joined Western Digital working on digital and analog circuit design. For his graduate work, he was in the Berkeley Sensor \& Actuator Center at the University of California at Berkeley specializing in MEMS, with a dissertation on micromachined hot-filament vacuum devices. From 1997 to 2000, he worked at NovaSensor developing micromachined allsilicon plate valves using a novel thermal actuator. From 2000 to 2003, he was with Agilent Technologies performing R\&D on high-Q variable capacitors and an optical switch. He is presently a MEMS consultant and is also a Lecturer in MEMS short courses run by the University of California at Berkeley Extension. $\mathrm{He}$ in an inventor on several patents and has authored or coauthored a number of papers and a book chapter related to MEMS. 


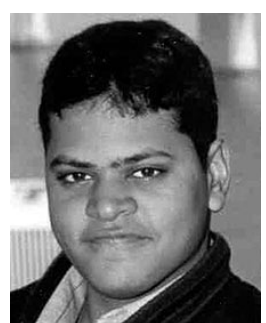

Kishan Gupta (S'01) received the B.S. degree in electrical engineering and computer sciences with highest honors from the University of California at Berkeley, in 2003. He is presently a post-baccalaureate student at San Jose State University, CA.

While pursuing the B.S. degree, he worked as a summer student at Agilent Technologies and at National Semiconductor. As an undergraduate, he performed research at the Berkeley Sensor \& Actuator Center at the University of California at Berkeley. His research interests include optical and biological

MEMS

Mr. Gupta is a Member of Tau Beta Pi and has held various officer positions for the Mu chapter of Eta Kappa Nu. He received an International Engineering Consortium William L. Everitt Student Award for Excellence and an honorable mention for the 2003 Eta Kappa Nu Alton B. Zerby and Carl T. Koerner Outstanding Electrical Engineering Student Award.

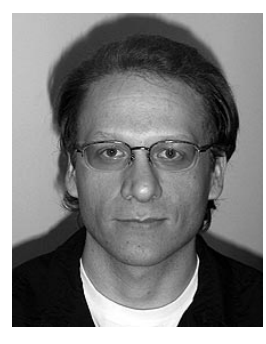

Matthew Wasilik received the B.S. degree in mechanical engineering from the University of Colorado in 1995. After working in industry for several years as a mechanical design engineer, he received the M.S. degree in electrical engineering from Colorado University, Boulder, in spring 2000.

He has authored or coauthored a number of papers relating to MEMS, and currently works as a Senior Development Engineer for the Berkeley Sensor \& Actuator Center at the University of California at Berkeley. 